\begin{tabular}{|c|l|}
\hline Title & Spectral A nalysis of an Effective Hamiltonian in Nonrelativistic Quantum Electrodynamics \\
\hline Author(s) & A rai, A sao \\
\hline Citation & $\begin{array}{l}\text { Annales Henri Poincaré, 12(1), 119-152 } \\
\text { https://doi.org/10.1007/300023-010-0071-2 }\end{array}$ \\
\hline Issue Date & 2011-01-11 \\
\hline Doc URL & http://hdl.handle.net/2115/44853 \\
\hline Rights & The original publication is available at www.springerlink.com \\
\hline Type & article (author version) \\
\hline File Information & EFFH-rev.pdf \\
\hline
\end{tabular}

Instructions for use 


\title{
Spectral Analysis of an Effective Hamiltonian in Nonrelativistic Quantum Electrodynamics
}

\author{
Asao Arai \\ Department of Mathematics, Hokkaido University \\ Sapporo 060-0810 \\ Japan \\ E-mail: arai@math.sci.hokudai.ac.jp
}

\begin{abstract}
We investigate spectral properties of an effective Hamiltonian which is obtained as a scaling limit of the Pauli-Fierz model in nonrelativistic quantum electrodynamics. The Lamb shift of a hydrogen-like atom is derived as the lowest order approximation (in the fine structure constant) of an energy level shift of the effective Hamiltonian.
\end{abstract}

Keywords: effective Hamiltonian, Lamb shift, nonrelativistic quantum electrodynamics, spectrum

Mathematics Subject Classification: 47N50, 81Q10, 81Q15, 81V10

\section{Introduction}

We consider the quantum system of a nonrelativistic spinless charged particle with mass $m>0$ and charge $q \in \mathbb{R} \backslash\{0\}$ in the $d$-dimensional Euclidean vector space $\mathbb{R}^{d}(d \geq 2)$ under the influence of a scalar potential $V: \mathbb{R}^{d} \rightarrow \mathbb{R}$, Borel measurable and almost everywhere finite with respect to the Lebesgue measure on $\mathbb{R}^{d}$. As is well known, a standard Hamiltonian of such a quantum system is given by the Schrödinger operator

$$
H:=-\frac{\hbar^{2}}{2 m} \Delta+V
$$

on $L^{2}\left(\mathbb{R}^{d}\right)$, where $\hbar:=h / 2 \pi$ ( $h$ is the Planck constant) and $\Delta$ is the generalized Laplacian on $L^{2}\left(\mathbb{R}^{d}\right)$. From a quantum field theoretical point of view, however, the operator $H$ is an approximate Hamiltonian, because the charged particle interacts with the quantum radiation field too. To incorporate this interaction, one has to extend the theoretical framework to nonrelativistic quantum electrodynamics (QED), a quantum theory describing nonrelativistic charged particles interacting with the quantum radiation field (for reviews on recent developments of mathematical theory of nonrelativistic QED, see, e.g., [6, 14]). 
Instead of doing a full analysis in the framework of nonrelativistic QED, one may take an intermediate way to make corrections due to the interaction of the charged particle with the quantum radiation field. This kind of approach (heuristic) was first given by Welton [15], based on the following physical picture: The position of the charged particle should have fluctuations caused by the interaction with the quantum radiation field. Then the fluctuations would change the potential $V$ and this change may give rise to observable effects due to the existence of the quantum radiation field. Indeed, e.g., the Lamb shift of the hydrogen atom - the energy level shift between $2 S_{1 / 2}$-state and $2 P_{1 / 2}$-state due to the interaction with the quantum radiation field (for a pedagogical physical explanation, see, e.g., [3, pp.57-60]) - can be heuristically explained in this way [15] (cf. also [2] for a formal perturbation theoretical treatment).

A mathematically rigorous structure behind the heuristic arguments of Welton [15] was formulated in the paper [1] where the author considers a scaling limit of a Hamiltonian in nonrelativistic QED and derives an effective potential which is a deformation of the original potential $V$ and may "reflect" or "include" effects due to the interaction of the charged particle with the quantum radiation field (for further developments of scaling limits in nonrelativistic QED, see [5]). The model used in [1], which is a simplified version of the full Pauli-Fierz model [9] with a mass renormalization, contains a Borel measurable function $\omega: \mathbb{R}^{d} \rightarrow[0, \infty) ; \mathbb{R}^{d} \ni \mathbf{k} \mapsto \omega(\mathbf{k})$ (a one-photon dispersion relation) and a real tempered distribution $\rho \neq 0$ on $\mathbb{R}^{d}$ satisfying

$$
\int_{\mathbb{R}^{d}} \frac{|\hat{\rho}(\mathbf{k})|^{2}}{\omega(\mathbf{k})^{3}} d \mathbf{k}<\infty, \quad \int_{\mathbb{R}^{d}} \frac{|\hat{\rho}(\mathbf{k})|^{2}}{\omega(\mathbf{k})} d \mathbf{k}<\infty
$$

where $\hat{\rho}$ denotes the Fourier transform of $\rho$ :

$$
\hat{\rho}(\mathbf{k}):=\frac{1}{(2 \pi)^{d / 2}} \int_{\mathbb{R}^{d}} e^{-i \mathbf{k x}} \rho(\mathbf{x}) d \mathbf{x},
$$

physically playing a role of a photon momentum cutoff function.

Let

$$
\lambda_{q}:=\frac{(d-1)}{4 d}\left(\frac{\hbar}{m c}\right)^{2} \frac{q^{2}}{\hbar c} \int_{\mathbb{R}^{d}} \frac{|\hat{\rho}(\mathbf{k})|^{2}}{\omega(\mathbf{k})^{3}} d \mathbf{k}
$$

with $c$ being the speed of light, and, for each $\lambda>0$,

$$
V_{\lambda}(\mathbf{x}):=\frac{1}{(4 \pi \lambda)^{d / 2}} \int_{\mathbb{R}^{d}} e^{-|\mathbf{x}-\mathbf{y}|^{2} / 4 \lambda} V(\mathbf{y}) d \mathbf{y}, \quad \mathbf{x}=\left(x_{1}, \cdots, x_{d}\right) \in \mathbb{R}^{d},
$$

where we assume that

$$
\int_{\mathbb{R}^{d}} e^{-t|\mathbf{x}-\mathbf{y}|^{2}}|V(\mathbf{y})| d \mathbf{y}<\infty, \quad \forall \mathbf{x} \in \mathbb{R}^{d}, \forall t>0
$$

Then the effective potential, denoted $V_{\text {eff }}: \mathbb{R}^{d} \rightarrow \mathbb{R}$, has the following form [1]:

$$
V_{\mathrm{eff}}=V_{\lambda_{q}} \text {. }
$$


Thus, to justify the interpretation mentioned above, one has to investigate spectral properties of the effective Hamiltonian

$$
H_{\mathrm{eff}}:=-\frac{\hbar^{2}}{2 m} \Delta+V_{\mathrm{eff}}
$$

and compare them with experimental results. This is one of the motivations for the present work.

The charge $q$ in $\lambda_{q}$ is originally a perturbation parameter in the Pauli-Fierz model, representing the coupling constant of the charged particle with the quantum radiation field [1]. But we note that $\left\{\lambda_{q} \mid q \in \mathbb{R} \backslash\{0\}\right\}=(0, \infty)$ and that taking the limit $\lambda_{q} \rightarrow 0$ is equivalent to $q \rightarrow 0$. Hence we are led to analyze the operator

$$
H_{\lambda}:=-\frac{\hbar^{2}}{2 m} \Delta+V_{\lambda}, \quad \lambda>0
$$

instead of $H_{\text {eff }}$. Then one obviously has

$$
H_{\mathrm{eff}}=H_{\lambda_{q}}
$$

Moreover, we have, in the distribution sense,

$$
\lim _{\lambda \rightarrow 0} \frac{1}{(4 \pi \lambda)^{d / 2}} e^{-|\mathbf{x}-\mathbf{y}|^{2} / 4 \lambda}=\delta(\mathbf{x}-\mathbf{y})
$$

the Dirac delta distribution on $\mathbb{R}^{d} \times \mathbb{R}^{d}$. This suggests that $V_{\lambda}$ is a perturbation of $V$ with perturbation parameter $\lambda$. Indeed, e.g., if $V$ is bounded and continuous on $\mathbb{R}^{d}$, then $\lim _{\lambda \rightarrow 0} V_{\lambda}(\mathbf{x})=V(\mathbf{x}), \forall \mathbf{x} \in \mathbb{R}^{d}$ (see also Lemma 2.1). But, in general, $H_{\lambda}$ is not necessarily a regular perturbation of $H$. This makes the mathematical analysis of $H_{\lambda}$ nontrivial. We also note that $V_{\lambda}$ is a Gauss transform of $V$. This kind of perturbation of $V$ may be mathematically interesting in its own right too.

By abuse of terminology, we also call $V_{\lambda}$ (resp. $H_{\lambda}$ ) an effective potential (resp. Hamiltonian) for $V$ (resp. $H$ ).

The present paper is organized as follows. In Section 2 we first investigate properties of the effective potential $V_{\lambda}$. Then we consider the effective Hamiltonian $H_{\lambda}$ with the space dimension $d$ general. For some classes of potentials $V$, we prove a stability theorem for a discrete eigenvalue of $H_{\lambda}$ (Theorems 2.5-2.7). We also establish a stability theorem for the essential spectrum of $H_{\lambda}$ for a class of potentials $V$ (Theorem 2.10). In Section 3 we specialize the space dimension $d$ to $d=3$ and consider the stability of properties of $V$ under the change $V \mapsto V_{\lambda}$. Section 4 is concerned with the infiniteness or the finiteness of discrete eigenvalues of a self-adjoint extension $\widetilde{H}_{\lambda}$ of $H_{\lambda}$. Section 5 is devoted to analysis of $H_{\lambda}$ with $V$ spherically symmetric in $\mathbb{R}^{3}$. In this case $V_{\lambda}$ is also spherically symmetric. We prove a stability theorem for a discrete eigenvalue of $H_{\lambda}$ (Theorem 5.4, Corollary 5.5). Moreover, the reduction of $H_{\lambda}$ to the closed subspaces which naturally appear in the polar coordinate representation in $\mathbb{R}^{3}$ is discussed (Corollary 5.6). In the last section we apply the results established in Section 5 to the effective Hamiltonian of a hydrogenlike atom - an atom consisting of an electron and a nucleus with charge $Z e(Z \in \mathbb{N}$ and $e>0$ is the fundamental charge) - and show that the Lamb shift can be derived as the 
lowest order term in the asymptotic expansion of an energy level shift of $H_{\lambda_{-e}}$ as $e \rightarrow 0^{1}$. The present paper has an appendix in which some general aspects of a (not necessarily regular) perturbation theory for self-adjoint operators are presented.

\section{The Effective Hamiltonian in General Cases}

In this section, we consider the effective Hamiltonian $H_{\lambda}$ with $d$ general. The physical case $d=3$ is discussed later in detail (Sections 3-6). To analyze properties of $H_{\lambda}$, we first need to know properties of the effective potential $V_{\lambda}$.

\subsection{Properties of the effective potential}

Let

$$
G_{\lambda}(\mathbf{x}):=\frac{1}{(4 \pi \lambda)^{d / 2}} e^{-\mathbf{x}^{2} / 4 \lambda}, \quad \mathbf{x} \in \mathbb{R}^{d}, \quad \lambda>0,
$$

a Gaussian function. By (1.4), one can write

$$
V_{\lambda}=G_{\lambda} * V
$$

where

$$
(f * g)(\mathbf{x}):=\int_{\mathbb{R}^{d}} f(\mathbf{x}-\mathbf{y}) g(\mathbf{y}) d \mathbf{y}
$$

the convolution of functions $f$ and $g$ on $\mathbb{R}^{d}$, provided that the integral on the right hand side exists. Hence $V_{\lambda}$ is the Gauss transform of $V$ with the Gaussian function $G_{\lambda}$.

Note that, if $V$ is in $L^{2}\left(\mathbb{R}^{d}\right)$, then

$$
V_{\lambda}=e^{\lambda \Delta} V, \quad \forall \lambda \geq 0 .
$$

For $p \in[1, \infty)$ or $p=\infty$ and $f \in L^{p}\left(\mathbb{R}^{d}\right)$, we denote by $\|f\|_{p}$ the $L^{p}$-norm of $f$ :

$$
\|f\|_{p}:=\left(\int_{\mathbb{R}^{d}}|f(\mathbf{x})|^{p} d \mathbf{x}\right)^{1 / p}(1 \leq p<\infty), \quad\|f\|_{\infty}:=\operatorname{ess.sup}_{\mathbf{x} \in \mathbb{R}^{d}}|f(\mathbf{x})|,
$$

where ess.sup means essential supremum.

Lemma 2.1 If $V \in L^{p}\left(\mathbb{R}^{d}\right)(1 \leq p \leq \infty)$, then (1.5) holds and $V_{\lambda} \in L^{p}\left(\mathbb{R}^{d}\right)$ for all $\lambda>0$ with

$$
\left\|V_{\lambda}\right\|_{p} \leq\|V\|_{p}
$$

Moreover, the following (i) and (ii) holds:

(i) If $1 \leq p<\infty$, then

$$
\lim _{\lambda \rightarrow 0}\left\|V_{\lambda}-V\right\|_{p}=0
$$

\footnotetext{
${ }^{1}$ An approach to energy level shift of the hydrogen-like atom using the full Pauli-Fierz Hamiltonian has been given in [4]. But it is completely different from our one.
} 
(ii) If $V \in L^{\infty}\left(\mathbb{R}^{d}\right)$ and $V$ is uniformly continuous on $\mathbb{R}^{d}$, then

$$
\lim _{\lambda \rightarrow 0}\left\|V_{\lambda}-V\right\|_{\infty}=0
$$

Proof. These are well known facts ( see, e.g., [7, Theorem 5.7]).

It may be convenient from perturbation theoretical point of view to set

$$
V_{0}:=V
$$

Then Lemma 2.1 shows that the mapping: $[0, \infty) \ni \lambda \mapsto V_{\lambda}$ is strongly continuous at $\lambda=0$ respectively in $L^{p}\left(\mathbb{R}^{d}\right)(1 \leq p<\infty)$ and in the space of uniformly continuous, bounded functions on $\mathbb{R}^{d}$.

We denote by $C^{1}\left(\mathbb{R}^{d}\right)$ the set of continuously differentiable functions on $\mathbb{R}^{d}$. For $f \in C^{1}\left(\mathbb{R}^{d}\right)$, we denote its gradient by $\nabla f$ :

$$
\nabla f:=\left(\partial_{1} f, \cdots, \partial_{d} f\right)
$$

with $\partial_{j} f:=\partial f / \partial x_{j}, j=1, \cdots, d$. Let

$$
C_{\mathrm{b}}^{1}\left(\mathbb{R}^{d}\right):=\left\{f \in C^{1}\left(\mathbb{R}^{d}\right) \mid \partial_{j} f \in L^{\infty}\left(\mathbb{R}^{d}\right), j=1, \cdots, d\right\},
$$

the set of continuously differentiable functions with all the partial derivatives bounded on $\mathbb{R}^{d}$, and

$$
\|\nabla f\|_{\infty}:=\sup _{\mathbf{x} \in \mathbb{R}^{d}}|\nabla f(\mathbf{x})|
$$

Lemma 2.2 Let $V \in C_{\mathrm{b}}^{1}\left(\mathbb{R}^{d}\right)$. Then, (1.5) holds and

$$
\left\|V_{\lambda}-V\right\|_{\infty} \leq \Gamma_{d} \sqrt{\lambda}\|\nabla V\|_{\infty}
$$

where

$$
\Gamma_{d}:=2 \pi^{-d / 2} \int_{\mathbb{R}^{d}} e^{-|\mathbf{z}|^{2}}|\mathbf{z}| d \mathbf{z}<\infty
$$

Proof. We have for all $\mathbf{a}, \mathbf{x} \in \mathbb{R}^{d}$ and $\alpha \geq 0$

$$
V(\mathbf{a}+\alpha \mathbf{x})-V(\mathbf{a})=\int_{0}^{\alpha} \mathbf{x}(\nabla V)(\mathbf{a}+t \mathbf{x}) d t
$$

Hence

$$
|V(\mathbf{a}+\mathbf{x})-V(\mathbf{a})| \leq|\mathbf{x}||| \nabla V \|_{\infty} .
$$

In particular, taking $\mathbf{a}=\mathbf{0}$, we have

$$
|V(\mathbf{x})| \leq|V(\mathbf{0})|+|\mathbf{x}||| \nabla V \|_{\infty} .
$$

This implies that (1.5) holds. 
By the change of variable: $\mathbf{y} \mapsto \mathbf{z}=(\mathbf{y}-\mathbf{x}) / 2 \sqrt{\lambda}$, we have

$$
V_{\lambda}(x)=\pi^{-d / 2} \int_{\mathbb{R}^{d}} e^{-|\mathbf{z}|^{2}} V(\mathbf{x}+2 \sqrt{\lambda} \mathbf{z}) d \mathbf{z} .
$$

By this formula and the fact that

$$
\pi^{-d / 2} \int_{\mathbb{R}^{d}} e^{-|\mathbf{z}|^{2}} d \mathbf{z}=1
$$

we obtain

$$
V_{\lambda}(\mathbf{x})-V(\mathbf{x})=\pi^{-d / 2} \int_{\mathbb{R}^{d}} e^{-|\mathbf{z}|^{2}}[V(\mathbf{x}+2 \sqrt{\lambda} \mathbf{z})-V(\mathbf{x})] d \mathbf{z} .
$$

By (2.14), we have

$$
|V(\mathbf{x}+2 \sqrt{\lambda} \mathbf{z})-V(\mathbf{x})| \leq 2 \sqrt{\lambda}|\mathbf{z}|\|\nabla V\|_{\infty}
$$

Thus (2.11) follows.

From a regular perturbation theoretical point of view, it would be natural to ask if there is a class of potentials $V$ such that the mapping : $\lambda \mapsto V_{\lambda}$ can be extended to an analytic vector-valued function in a suitable topology. A class of such potentials $V$ is given in the following lemma:

Lemma 2.3 Suppose that $\hat{V} \in C_{0}^{\infty}\left(\mathbb{R}^{d}\right)$ (the set of infinitely differentiable functions on $\mathbb{R}^{d}$ with compact support). Then, $V_{\lambda} \in \mathcal{S}\left(\mathbb{R}^{d}\right)$ (the Schwartz space of rapidly decreasing functions on $\mathbb{R}^{d}$ ) for all $\lambda \geq 0$ and

$$
V_{\lambda}(\mathbf{x})=\sum_{n=0}^{\infty} \frac{\left(\Delta^{n} V\right)(\mathbf{x})}{n !} \lambda^{n}, \quad \mathbf{x} \in \mathbb{R}^{d}, \lambda \geq 0
$$

uniformly in $\mathbf{x}$ on $\mathbb{R}^{d}$, where the series on the right hand side is absolutely convergent.

Proof. By the present assumption, there exists a constant $K>0$ such that $\hat{V}(\mathbf{k})=$ $0,|\mathbf{k}| \geq K$. By Fourier analysis, we have

$$
V_{\lambda}(\mathbf{x})=\frac{1}{(2 \pi)^{d / 2}} \int_{|\mathbf{k}| \leq K} e^{i \mathbf{k x}} e^{-\lambda \mathbf{k}^{2}} \hat{V}(\mathbf{k}) d \mathbf{k}
$$

The function $: \mathbf{k} \mapsto e^{-\lambda \mathbf{k}^{2}} \hat{V}(\mathbf{k})$ is in $C_{0}^{\infty}\left(\mathbb{R}^{d}\right)$ and hence in $\mathcal{S}\left(\mathbb{R}^{d}\right)$. Therefore $V_{\lambda}$ is in $\mathcal{S}\left(\mathbb{R}^{d}\right)$. We have

$$
\left|\Delta^{n} V(\mathbf{x})\right| \leq \frac{1}{(2 \pi)^{d / 2}} K^{2 n}\|\hat{V}\|_{1} .
$$

Hence $\sum_{n=0}^{\infty}\left(\Delta^{n} V\right)(\mathbf{x}) \lambda^{n} / n$ ! converges absolutely with

$$
\sum_{n=0}^{\infty} \frac{\left|\left(\Delta^{n} V\right)(\mathbf{x}) \lambda^{n}\right|}{n !} \leq \frac{1}{(2 \pi)^{d / 2}} e^{K^{2} \lambda}\|\hat{V}\|_{1} .
$$


Let $S_{N}(\mathbf{x}):=\sum_{n=0}^{N}\left(\Delta^{n} V\right)(\mathbf{x}) \lambda^{n} / n !, N \in \mathbb{N}$. Then we have

$$
\begin{aligned}
\left|V_{\lambda}(\mathbf{x})-S_{N}(\mathbf{x})\right| & \leq \frac{1}{(2 \pi)^{d / 2}} \int_{|\mathbf{k}| \leq K}\left|e^{-\lambda \mathbf{k}^{2}}-\sum_{n=0}^{N} \frac{\left(-\mathbf{k}^{2}\right)^{n} \lambda^{n}}{n !}\right||\hat{V}(\mathbf{k})| d \mathbf{k} \\
& \leq \frac{1}{(2 \pi)^{d / 2}} \sum_{n=N+1}^{\infty} \frac{\left(\lambda K^{2}\right)^{n}}{n !}\|\hat{V}\|_{1} .
\end{aligned}
$$

Hence $\lim _{N \rightarrow \infty} \sup _{\mathbf{x} \in \mathbb{R}^{d}}\left|V_{\lambda}(\mathbf{x})-S_{N}(\mathbf{x})\right|=0$.

\subsection{Existence of discrete eigenvalues of the effective Hamilto- nian}

We want to find classes of potentials $V$ such that $H_{\lambda}(\lambda>0)$ has an eigenvalue if the unperturbed operator $H$ has an eigenvalue. In this subsection, we consider only simple classes of such $V$.

In what follows, for a Hilbert space $\mathcal{H}$, we denote its inner product and norm by $\langle\cdot, \cdot\rangle$ (antilinear in the first variable and linear in the second one) and $\|\cdot\|$ respectively. The domain (resp. range) of a linear operator $A$ on $\mathcal{H}$ is denoted $D(A)(\operatorname{resp}$. $\operatorname{Ran}(A))$. We denote the spectrum and the resolvent set of $A$ by $\sigma(A)$ and $\rho(A)$ respectively. If $A$ is bounded, we denote its operator norm by $\|A\|$.

For a self-adjoint operator $A$, we denote its spectral measure by $E_{A}$.

The next lemma is a key fact in our theory below:

Lemma 2.4 Let $\left\{A_{\lambda}\right\}_{\lambda>0}$ and $A$ be self-adjoint operators on a Hilbert space. Suppose that $A_{\lambda}$ converges to $A$ in the norm resolvent sense as $\lambda \rightarrow 0$ :

$$
\lim _{\lambda \rightarrow 0}\left\|\left(A_{\lambda}-z\right)^{-1}-(A-z)^{-1}\right\|=0, \quad z \in \mathbb{C} \backslash \mathbb{R} .
$$

Let $a, b \in \rho(A) \cap \mathbb{R}, a<b$. Then, there exists a constant $r>0$ such that

$$
\operatorname{dim} \operatorname{Ran}\left(E_{A_{\lambda}}((a, b))\right)=\operatorname{dim} \operatorname{Ran}\left(E_{A}((a, b))\right), \quad \lambda \in(0, r) .
$$

Proof. By the present assumption and [10, Theorem VIII.23-(b)] (cf. also [10, Theorem VIII.19]), we have

$$
\lim _{\lambda \rightarrow 0}\left\|E_{A_{\lambda}}((a, b))-E_{A}((a, b))\right\|=0 .
$$

Hence there exists a constant $r>0$ such that

$$
\left\|E_{A_{\lambda}}((a, b))-E_{A}((a, b))\right\|<1, \quad \lambda \in(0, r) .
$$

This inequality and a general fact [12, p.14, Lemma] imply (2.17).

Going back to the effective Hamiltonian $H_{\lambda}$, we first consider the case where $V$ is in $L^{\infty}\left(\mathbb{R}^{d}\right)$. In this case, $H$ is self-adjoint with $D(H)=D(\Delta)$. By Lemma $2.1, V_{\lambda}$ is in $L^{\infty}\left(\mathbb{R}^{d}\right)$. Hence $H_{\lambda}$ also is self-adjoint with $D\left(H_{\lambda}\right)=D(\Delta)$. The next theorem shows the stability of an eigenvalue of $H$ under the change of $V$ to $V_{\lambda}$ : 
Theorem 2.5 Let $V$ be in $L^{\infty}\left(\mathbb{R}^{d}\right)$ and uniformly continuous on $\mathbb{R}^{d}$. Let $a, b \in \mathbb{R}$ with $a<b$. Suppose that $H$ has an isolated eigenvalue $E_{0}$ in the open interval $(a, b)$ with multiplicity $m\left(E_{0}\right)$ and that $\sigma(H) \cap(a, b)=\left\{E_{0}\right\}$. Then, there exists a constant $r>0$ such that, for all $\lambda \in(0, r), H_{\lambda}$ has exactly $m\left(E_{0}\right)$ discrete eigenvalues in $(a, b)$, counting multiplicities, and $\sigma\left(H_{\lambda}\right) \cap(a, b)$ consists of only these eigenvalues. In particular, if $m\left(E_{0}\right)=1$, then $H_{\lambda}$ with $\lambda \in(0, r)$ has a unique isolated simple eigenvalue $E_{\lambda}$ in $(a, b)$ and

$$
\lim _{\lambda \rightarrow 0} E_{\lambda}=E_{0}
$$

Proof. Let $z \in \mathbb{C} \backslash \mathbb{R}$. Then

$$
\left(H_{\lambda}-z\right)^{-1}-(H-z)^{-1}=\left(H_{\lambda}-z\right)^{-1}\left(V-V_{\lambda}\right)(H-z)^{-1} .
$$

Hence

$$
\left\|\left(H_{\lambda}-z\right)^{-1}-(H-z)^{-1}\right\| \leq \frac{1}{|\operatorname{Im} z|}\left\|V-V_{\lambda}\right\|_{\infty}\left\|(H-z)^{-1}\right\|,
$$

where $\operatorname{Im} z$ denotes the imaginary part of $z$. By Lemma 2.1-(ii), for every $\varepsilon>0$, there exists a constant $r_{\varepsilon}>0$ such that, for all $\lambda \in\left(0, r_{\varepsilon}\right),\left\|V-V_{\lambda}\right\|_{\infty}<\varepsilon$. Hence

$$
\left\|\left(H_{\lambda}-z\right)^{-1}-(H-z)^{-1}\right\|<\varepsilon \frac{\left\|(H-z)^{-1}\right\|}{|\operatorname{Im} z|}, \quad \lambda \in\left(0, r_{\varepsilon}\right) .
$$

Thus $H_{\lambda}$ converges to $H$ in the norm resolvent sense as $\lambda \rightarrow 0$. Hence, by Lemma 2.4 , there exists a constant $r>0$ such that, for all $\lambda \in(0, r), \operatorname{dim} \operatorname{Ran}\left(E_{H_{\lambda}}((a, b))\right)=$ $\operatorname{dim} \operatorname{Ran}\left(E_{H}((a, b))\right)=m\left(E_{0}\right)$. Thus $H_{\lambda}$ has exactly $m\left(E_{0}\right)$ discrete eigenvalues in $(a, b)$, counting multiplicities, and $\sigma\left(H_{\lambda}\right) \cap(a, b)$ consist of only these eigenvalues.

Suppose that $m\left(E_{0}\right)=1$ and $\Omega_{0}$ is a normalized eigenvector of $H$ with eigenvalue $E_{0}$ : $H \Omega_{0}=E_{0} \Omega_{0},\left\|\Omega_{0}\right\|=1$. Then, by the preceding result, $H_{\lambda}$ with $\lambda \in(0, r)$ has exactly one eigenvalue $E_{\lambda}$ in $(a, b)$ with $\sigma\left(H_{\lambda}\right) \cap(a, b)=\left\{E_{\lambda}\right\}$. We set $P_{\lambda}:=E_{H_{\lambda}}((a, b))$ and $P:=E_{H}((a, b))$. Then $P \Omega_{0}=\Omega_{0}$ and $H_{\lambda} P_{\lambda} \Omega_{0}=E_{\lambda} P_{\lambda} \Omega_{0}$. Taking the inner product of this equation with $\Omega_{0}$, we have

$$
E_{\lambda}\left\langle\Omega_{0}, P_{\lambda} \Omega_{0}\right\rangle=E_{0}\left\langle\Omega_{0}, P_{\lambda} \Omega_{0}\right\rangle+\left\langle\Omega_{0},\left(V_{\lambda}-V\right) \Omega_{0}\right\rangle+\left\langle\left(V_{\lambda}-V\right) \Omega_{0},\left(P_{\lambda}-P\right) \Omega_{0}\right\rangle .
$$

By (2.18) with $A_{\lambda}=H_{\lambda}$ and $A=H$, we have $\left\|P_{\lambda}-P\right\|<1, \lambda \in(0, r)$. Hence it follows that $\left\|P_{\lambda} \Omega_{0}\right\|>0$, which implies that $\left\langle\Omega_{0}, P_{\lambda} \Omega_{0}\right\rangle>0$. Therefore we obtain

$$
E_{\lambda}=E_{0}+\frac{\left\langle\Omega_{0},\left(V_{\lambda}-V\right) \Omega_{0}\right\rangle}{\left\langle\Omega_{0}, P_{\lambda} \Omega_{0}\right\rangle}+\frac{\left\langle\left(V_{\lambda}-V\right) \Omega_{0},\left(P_{\lambda}-P\right) \Omega_{0}\right\rangle}{\left\langle\Omega_{0}, P_{\lambda} \Omega_{0}\right\rangle} .
$$

Note that

$$
\left\langle\Omega_{0}, P_{\lambda} \Omega_{0}\right\rangle=1+\left\langle\Omega_{0},\left(P_{\lambda}-P\right) \Omega_{0}\right\rangle=1+o(1) \quad(\lambda \rightarrow 0) .
$$

Thus (2.19) holds.

We next consider the case where $V$ is in $C_{\mathrm{b}}^{1}\left(\mathbb{R}^{d}\right)$ and bounded below. Then, by a general theorem [11, Theorem X.28], $H$ is essentially self-adjoint on $C_{0}^{\infty}\left(\mathbb{R}^{d}\right)$. We denote the closure of $H$ by $\bar{H}$. By Lemma $2.2, V_{\lambda}-V$ is bounded on $\mathbb{R}^{d}$. Hence $H_{\lambda}$ is essentially self-adjoint on $C_{0}^{\infty}\left(\mathbb{R}^{d}\right)$. For $t \geq 0$, we define $V_{t}^{(1)}: \mathbb{R}^{d} \rightarrow \mathbb{R}$ by

$$
V_{t}^{(1)}(\mathbf{x}):=\frac{1}{\pi^{d / 2}} \int_{\mathbb{R}^{d}} e^{-|\mathbf{y}|^{2}} \mathbf{y}(\nabla V)(\mathbf{x}+t \mathbf{y}) d \mathbf{y} .
$$


Theorem 2.6 Let $V$ be in $C_{\mathrm{b}}^{1}\left(\mathbb{R}^{d}\right)$ and bounded below. Let $a, b \in \rho(\bar{H}) \cap \mathbb{R}(a<b)$. Suppose that $\bar{H}$ has an isolated eigenvalue $E_{0}$ in the open interval $(a, b)$ with multiplicity $m\left(E_{0}\right)$ and $\sigma(\bar{H}) \cap(a, b)=\left\{E_{0}\right\}$. Then, there exists a constant $r>0$ such that, for all $\lambda \in(0, r), \bar{H}_{\lambda}$ has exactly $m\left(E_{0}\right)$ discrete eigenvalues in $(a, b)$, counting multiplicities, and $\sigma\left(\bar{H}_{\lambda}\right) \cap(a, b)$ consists of only these eigenvalues. In particular, if $m\left(E_{0}\right)=1$, then $\bar{H}_{\lambda}$ with $\lambda \in(0, r)$ has a unique isolated simple eigenvalue $E_{\lambda}$ in $(a, b)$ with $\sigma\left(\bar{H}_{\lambda}\right) \cap(a, b)=\left\{E_{\lambda}\right\}$ and

$$
E_{\lambda}=E_{0}+\int_{0}^{2 \sqrt{\lambda}}\left\langle\Omega_{0}, V_{t}^{(1)} \Omega_{0}\right\rangle d t+o(\sqrt{\lambda}) \quad(\lambda \rightarrow 0),
$$

where $\Omega_{0}$ is a normalized eigenvector of $\bar{H}$ with eigenvalue $E_{0}: \bar{H} \Omega_{0}=E_{0} \Omega_{0},\left\|\Omega_{0}\right\|=1$.

Proof. Since we have Lemma 2.2, existence proof of eigenvalues of $\bar{H}_{\lambda}$ can be done in the same way as in the proof of Theorem 2.5. Thus we need only to prove (2.22). By (2.13) and (2.15), we have

$$
V_{\lambda}(\mathbf{x})-V(\mathbf{x})=\int_{0}^{2 \sqrt{\lambda}} V_{t}^{(1)}(\mathbf{x}) d t
$$

Hence

$$
\left\langle\Omega_{0},\left(V_{\lambda}-V\right) \Omega_{0}\right\rangle=\int_{\mathbb{R}^{d}}\left(\int_{0}^{2 \sqrt{\lambda}} V_{t}^{(1)}(\mathbf{x}) d t\right)\left|\Omega_{0}(\mathbf{x})\right|^{2} d \mathbf{x}
$$

Since we have

$$
\left|V_{t}^{(1)}(\mathbf{x})\right| \leq \frac{\Gamma_{d}}{2}\|\nabla V\|_{\infty}
$$

with $\Gamma_{d}$ given by (2.12), we can apply Fubini's theorem to the double integral on the right hand side to obtain

$$
\left\langle\Omega_{0},\left(V_{\lambda}-V\right) \Omega_{0}\right\rangle=\int_{0}^{2 \sqrt{\lambda}}\left\langle\Omega_{0}, V_{t}^{(1)} \Omega_{0}\right\rangle d t .
$$

By this formula and Lemma 2.2 together with (2.20), we can obtain (2.22).

In concluding this section, we consider the case where $V$ is a potential such that $\hat{V} \in C_{0}^{\infty}\left(\mathbb{R}^{d}\right)$. In this case we have Lemma 2.3. In particular, $H$ and $H_{\lambda}$ are self-adjoint with $D(H)=D\left(H_{\lambda}\right)=D(\Delta), \lambda>0$.

Theorem 2.7 Let $\hat{V} \in C_{0}^{\infty}\left(\mathbb{R}^{d}\right)$. Suppose that $H$ has an isolated eigenvalue $E_{0}$ with multiplicity $m\left(E_{0}\right)$. Then, there exists a constant $r>0$ such that, for all $\lambda \in(0, r)$, $H_{\lambda}$ has exactly $m\left(E_{0}\right)$ discrete eigenvalues $E^{(j)}(\lambda)\left(j=1, \cdots, m\left(E_{0}\right)\right)$ near $E_{0}$ and the spectrum of $H_{\lambda}$ in this region consists of only these eigenvalues. Moreover, each $E^{(j)}(\lambda)$ as a function of $\lambda$ has an analytic continuation to the disk $\{\lambda \in \mathbb{C}|| \lambda \mid<r\}$. In particular, if $m\left(E_{0}\right)=1$ and $H \Omega_{0}=E_{0} \Omega_{0},\left\|\Omega_{0}\right\|=1\left(\Omega_{0} \in D(H)\right)$, then $H_{\lambda}$ with $\lambda \in(0, r)$ has a unique isolated simple eigenvalue $E_{\lambda}$ near $E_{0}$ and

$$
E_{\lambda}=E_{0}+\frac{\sum_{n=0}^{\infty} a_{n}(\lambda)}{\sum_{n=0}^{\infty} b_{n}(\lambda)}
$$


with

$$
\begin{aligned}
a_{n}(\lambda) & :=\frac{(-1)^{n+1}}{2 \pi i} \sum_{\ell=0}^{\infty} \sum_{\ell_{1}, \cdots, \ell_{n}=0}^{\infty} \frac{\lambda^{\ell+\ell_{1}+\cdots+\ell_{n}}}{\ell ! \ell_{1} ! \cdots \ell_{n} !} \\
& \times \int_{\left|E-E_{0}\right|=\varepsilon}\left\langle\Omega_{0},\left(\Delta^{\ell} V\right)(H-E)^{-1}\left(\Delta^{\ell_{1}} V\right)(H-E)^{-1}\left(\Delta^{\ell_{2}} V\right)(H-E)^{-1}\right. \\
& \left.\times \cdots\left(\Delta^{\ell_{n}} V\right)(H-E)^{-1} \Omega_{0}\right\rangle d E \\
b_{n}(\lambda) & :=\frac{(-1)^{n+1}}{2 \pi i} \sum_{\ell_{1}, \cdots, \ell_{n}=0}^{\infty} \frac{\lambda^{\ell_{1}+\cdots+\ell_{n}}}{\ell_{1} ! \cdots \ell_{n} !} \\
& \times \int_{\left|E-E_{0}\right|=\varepsilon}\left\langle\Omega_{0},(H-E)^{-1}\left(\Delta^{\ell_{1}} V\right)(H-E)^{-1}\left(\Delta^{\ell_{2}} V\right)(H-E)^{-1}\right. \\
& \left.\times \cdots\left(\Delta^{\ell_{n}} V\right)(H-E)^{-1} \Omega_{0}\right\rangle d E
\end{aligned}
$$

where $\int_{\left|E-E_{0}\right|=\varepsilon} d E$ means the contour integral on the circle $\left|E-E_{0}\right|=\varepsilon$ with anti-clockwise orientation, $\varepsilon>0$ being sufficiently small.

Proof. By Lemma 2.3, we can define for all $\beta \in \mathbb{C}$ a linear operator $H(\beta)$ by

$$
H(\beta):=-\frac{\hbar^{2}}{2 m} \Delta+V(\beta)
$$

where $V(\beta):=\sum_{n=0}^{\infty}\left(\Delta^{n} V\right) \beta^{n} / n$ !. By Lemma $2.3, V(\beta)$ is bounded and analytic in $\beta$ in the uniform topology. For $\lambda>0$, we have $H(\lambda)=H_{\lambda}$. It is obvious that, for all $\beta \in \mathbb{C}, H(\beta)$ is a closed operator with $D(H(\beta))=D(\Delta)$ and, for all $f \in D(\Delta), H(\beta) f$ is a vector-valued analytic function of $\beta$. Since $\lim _{\beta \rightarrow 0}\|V(\beta)-V\|_{\infty}=0$, there exists a constant $\beta_{0}>0$ such that, for all $|\beta|<\beta_{0}, \rho(H(\beta)) \neq \emptyset$. Hence $\{H(\beta)\}_{|\beta|<\beta_{0}}$ is an analytic family of type (A) [12, p.16, Definition]. Thus we can apply a general theorem of regular perturbation theory [12, Theorem XII.13] to obtain the desired results.

\subsection{Essential spectrum of the effective Hamiltonian}

It is interesting to compare the essential spectrum of $H$, denoted $\sigma_{\text {ess }}(H)$, with that of $H_{\lambda}$. For this purpose, we recall a class of potentials $V$.

Let $p \geq 1$ and $\varepsilon>0$. We say that $V$ is in the set $L^{p}\left(\mathbb{R}^{d}\right)+L_{\varepsilon}^{\infty}\left(\mathbb{R}^{d}\right)$ if, for every $\varepsilon>0$, there exist functions $V_{1 \varepsilon} \in L^{p}\left(\mathbb{R}^{d}\right)$ and $V_{2 \varepsilon} \in L^{\infty}\left(\mathbb{R}^{d}\right)$ such that

$$
V=V_{1 \varepsilon}+V_{2 \varepsilon}, \quad\left\|V_{2 \varepsilon}\right\|_{\infty}<\varepsilon .
$$

Lemma 2.8 If $V$ is in $L^{p}\left(\mathbb{R}^{d}\right)+L_{\varepsilon}^{\infty}\left(\mathbb{R}^{d}\right)$, then so is $V_{\lambda}$ for all $\lambda>0$.

Proof. This follows from Lemma 2.1.

For a Borel measurable function $U: \mathbb{R}^{d} \rightarrow \mathbb{R}$, we define

$$
H_{U}:=-\frac{\hbar^{2}}{2 m} \Delta+U
$$


Lemma 2.9 Let $p=2$ for $d=1,2,3 ; p>d / 2$ for $d \geq 4$ and

$$
U \in L^{p}\left(\mathbb{R}^{d}\right)+L_{\varepsilon}^{\infty}\left(\mathbb{R}^{d}\right) .
$$

Then, $H_{U}$ is self-adjoint with $D\left(H_{U}\right)=D(\Delta)$ and bounded below. Moreover

$$
\sigma_{\mathrm{ess}}\left(H_{U}\right)=[0, \infty)
$$

Proof. Every $U \in L^{p}\left(\mathbb{R}^{d}\right)+L_{\varepsilon}^{\infty}\left(\mathbb{R}^{d}\right)$ with $p$ as above is infinitesimally small with respect to $\Delta$ (apply the proof of [11, Theorem X.15] in the case $d=1,2,3$ and [11, Theorem X.20] in the case $d \geq 4$ ). Hence, by the Kato-Rellich theorem [11, Theorem X.12], $H_{U}$ is self-adjoint and bounded below. Relation (2.27) follows from the fact that $\sigma_{\text {ess }}\left(H_{U}\right)=\sigma_{\text {ess }}(-\Delta)=[0, \infty)([12$, p.369, Problem 41] $)$.

For convenience, we set

$$
H_{0}:=H,
$$

so that we have a family $\left\{H_{\lambda}\right\}_{\lambda \geq 0}$ of symmetric operators indexed by the closed semiinfinite interval $[0, \infty)$.

The following theorem immediately follows from Lemmas 2.8 and 2.9:

Theorem 2.10 Let $p$ be as in Lemma 2.9 and $V \in L^{p}\left(\mathbb{R}^{d}\right)+L_{\varepsilon}^{\infty}\left(\mathbb{R}^{d}\right)$. Then, for all $\lambda \geq 0, H_{\lambda}$ is self-adjoint with $D\left(H_{\lambda}\right)=D(\Delta)$ and bounded below. Moreover

$$
\sigma_{\mathrm{ess}}\left(H_{\lambda}\right)=[0, \infty), \quad \forall \lambda \geq 0
$$

This theorem shows that, for the class of potentials $V$ specified there, the essential spectrum of $H_{0}=H$ is stable under the perturbation $V_{\lambda}-V$.

\section{Effective Potentials in Three Space Dimensions}

It is also interesting to consider the stability of the discrete spectrum of $H$, denoted $\sigma_{\text {disc }}(H)$, under the change $V \mapsto V_{\lambda}$. This section is a preliminary for this purpose.

From now on, we consider the physical case $d=3$. In this case, a typical class of potentials $V: \mathbb{R}^{3} \rightarrow \mathbb{R}$ is given by the Rollnik class $\mathcal{R}$ :

$$
V \in \mathcal{R} \stackrel{\text { def }}{\Longleftrightarrow} \int_{\mathbb{R}^{3} \times \mathbb{R}^{3}} \frac{|V(\mathbf{x})||V(\mathbf{y})|}{|\mathbf{x}-\mathbf{y}|^{2}} d \mathbf{x} d \mathbf{y}<\infty .
$$

An element $V$ in $\mathcal{R}$ is called a Rollnik potential. The following fact is well known (e.g., [13, Theorem I.15]):

Lemma 3.1 Let $V \geq 0$ and $V \in L^{1}\left(\mathbb{R}^{3}\right)$. Then, $V \in \mathcal{R}$ if and only if

$$
\int_{\mathbb{R}^{3}} \frac{|\hat{V}(\mathbf{k})|^{2}}{|\mathbf{k}|} d \mathbf{k}<\infty
$$


We introduce a subset of $\mathcal{R}$ :

$$
\mathcal{R}_{\mathrm{cs}}:=\{V \in \mathcal{R} \mid V \geq 0 \text { or } V \leq 0\},
$$

the set of Rollnik potentials with constant signature.

Lemma 3.2 Let $V \in \mathcal{R}_{\mathrm{cs}} \cap L^{1}\left(\mathbb{R}^{3}\right)$. Then, for all $\lambda>0, V_{\lambda} \in \mathcal{R}_{\mathrm{cs}} \cap L^{1}\left(\mathbb{R}^{3}\right)$.

Proof. By Lemma 2.1, we need only to show that $V_{\lambda} \in \mathcal{R}_{\mathrm{cs}}$. Let $V \in \mathcal{R}_{\mathrm{cs}}$ and $V \geq 0$. Then it is obvious that $V_{\lambda} \geq 0$. We have

$$
\hat{V}_{\lambda}(\mathbf{k})=e^{-\lambda \mathbf{k}^{2}} \hat{V}(\mathbf{k})
$$

Hence

$$
\int_{\mathbb{R}^{3}} \frac{\left|\hat{V}_{\lambda}(\mathbf{k})\right|^{2}}{|\mathbf{k}|} d \mathbf{k} \leq \int_{\mathbb{R}^{3}} \frac{|\hat{V}(\mathbf{k})|^{2}}{|\mathbf{k}|} d \mathbf{k}<\infty \quad \text { (by Lemma 3.1). }
$$

Hence, by Lemma 3.1, $V_{\lambda} \in \mathcal{R}$. Thus $V_{\lambda} \in \mathcal{R}_{\mathrm{cs}}$. Similarly one can prove this in the case $V \leq 0$.

Lemma 3.3 Let $V \in \mathcal{R} \cap L^{1}\left(\mathbb{R}^{3}\right)$. Then, for all $\lambda>0, V_{\lambda} \in \mathcal{R} \cap L^{1}\left(\mathbb{R}^{3}\right)$.

Proof. We have the decomposition $V=V_{+}-V_{-}$with $V_{+}(\mathbf{x}):=\max \{V(\mathbf{x}), 0\} \geq 0$ and $V_{-}(\mathbf{x}):=-\min \{V(\mathbf{x}), 0\} \geq 0$. It is easy to see that, if $V \in \mathcal{R} \cap L^{1}\left(\mathbb{R}^{3}\right)$, then $V_{ \pm} \in \mathcal{R}_{\mathrm{cs}} \cap L^{1}\left(\mathbb{R}^{3}\right)$. By this fact and Lemma 3.2, we obtain the desired result.

Lemma 3.4 Let $V \in \mathcal{R} \cap L^{1}\left(\mathbb{R}^{3}\right)+L^{\infty}\left(\mathbb{R}^{3}\right)$. Then, for all $\lambda>0, V_{\lambda} \in \mathcal{R} \cap L^{1}\left(\mathbb{R}^{3}\right)+$ $L^{\infty}\left(\mathbb{R}^{3}\right)$.

Proof. This follows from Lemmas 2.1 and 3.3.

Let $\mathcal{R}^{\prime}=\mathcal{R}$ or $\mathcal{R}_{\mathrm{cs}}$. We say that $V$ is in $\mathcal{R}^{\prime} \cap L^{1}\left(\mathbb{R}^{3}\right)+L^{\infty}\left(\mathbb{R}^{3}\right)_{\varepsilon}$ if, for every $\varepsilon>0$, there exist $V_{1 \varepsilon} \in \mathcal{R}^{\prime} \cap L^{1}\left(\mathbb{R}^{3}\right)$ and $V_{2 \varepsilon} \in L^{\infty}\left(\mathbb{R}^{3}\right)$ such that

$$
V=V_{1 \varepsilon}+V_{2 \varepsilon}, \quad\left\|V_{2 \varepsilon}\right\|_{\infty}<\varepsilon .
$$

Lemma 3.5 Let $V \in \mathcal{R}_{\mathrm{cs}} \cap L^{1}\left(\mathbb{R}^{3}\right)+L^{\infty}\left(\mathbb{R}^{3}\right)_{\varepsilon}$. Then, $V_{\lambda} \in \mathcal{R}_{\mathrm{cs}} \cap L^{1}\left(\mathbb{R}^{3}\right)+L^{\infty}\left(\mathbb{R}^{3}\right)_{\varepsilon}$ for all $\lambda>0$.

Proof. This follows from Lemmas 3.2 and 2.1.

Lemma 3.6 Let $V \in \mathcal{R} \cap L^{1}\left(\mathbb{R}^{3}\right)+L^{\infty}\left(\mathbb{R}^{3}\right)_{\varepsilon}$. Then, $V_{\lambda} \in \mathcal{R} \cap L^{1}\left(\mathbb{R}^{3}\right)+L^{\infty}\left(\mathbb{R}^{3}\right)_{\varepsilon}$ for all $\lambda>0$.

Proof. This follows from Lemma 3.4. by $V_{\lambda}$.

The following two lemmas show that some behaviors of $V$ near $|\mathbf{x}|=\infty$ are taken over 
Lemma 3.7 Assume (1.5). Suppose that there exist constants $a>0, \varepsilon>0$ and $R_{0}>0$ such that

$$
V(\mathbf{x}) \leq-\frac{a}{|\mathbf{x}|^{2-\varepsilon}}, \quad \forall|\mathbf{x}| \geq R_{0}
$$

Then, for each $\lambda>0$, there exist constants $a^{\prime}>0$ and $R_{0}^{\prime}>0$ such that

$$
V_{\lambda}(\mathbf{x}) \leq-\frac{a^{\prime}}{|\mathbf{x}|^{2-\varepsilon}}, \quad \forall|\mathbf{x}| \geq R_{0}^{\prime}
$$

Proof. We write

$$
V_{\lambda}=\frac{1}{(4 \pi \lambda)^{3 / 2}}\left(I_{1}+I_{2}\right)
$$

where

$$
I_{1}(\mathbf{x}):=\int_{|\mathbf{y}| \geq R_{0}} e^{-|\mathbf{x}-\mathbf{y}|^{2} / 4 \lambda} V(\mathbf{y}) d \mathbf{y}, \quad I_{2}(\mathbf{x}):=\int_{|\mathbf{y}|<R_{0}} e^{-|\mathbf{x}-\mathbf{y}|^{2} / 4 \lambda} V(\mathbf{y}) d \mathbf{y} .
$$

Using (3.5) and change of variables to polar coordinates, one can show that

$$
I_{1}(\mathbf{x}) \leq-\frac{4 \pi a \lambda}{|\mathbf{x}|} J_{1}(\mathbf{x})+\frac{4 \pi a \lambda}{|\mathbf{x}|} J_{2}(\mathbf{x}), \quad \mathbf{x} \neq \mathbf{0}
$$

where

$$
J_{1}(\mathbf{x}):=\int_{R_{0}}^{\infty} \frac{e^{-(r-|\mathbf{x}|)^{2} / 4 \lambda}}{r^{1-\varepsilon}} d r, \quad J_{2}(\mathbf{x}):=\int_{R_{0}}^{\infty} \frac{e^{-(r+|\mathbf{x}|)^{2} / 4 \lambda}}{r^{1-\varepsilon}} d r
$$

We have

$$
J_{1}(\mathbf{x})=\frac{1}{|\mathbf{x}|^{1-\varepsilon}} \int_{R_{0}-|\mathbf{x}|}^{\infty} \frac{e^{-s^{2} / 4 \lambda}}{\left(1+\frac{s}{|\mathbf{x}|}\right)^{1-\varepsilon}} d s .
$$

Let $0<\varepsilon<1$ and $|\mathbf{x}| \geq r_{0}>0$. Then

$$
J_{1}(\mathbf{x}) \geq \frac{1}{|\mathbf{x}|^{1-\varepsilon}} C_{1}
$$

with

$$
C_{1}:=\int_{R_{0}-r_{0}}^{\infty} \frac{e^{-s^{2} / 4 \lambda}}{\left(1+\frac{s}{r_{0}}\right)^{1-\varepsilon}} d s .
$$

On the other hand, if $1 \leq \varepsilon$ and $|\mathbf{x}| \geq r_{0}$, then

$$
J_{1}(\mathbf{x}) \geq \frac{1}{|\mathbf{x}|^{1-\varepsilon}} \int_{R_{0}-r_{0}}^{\infty}\left(1+\frac{s}{|\mathbf{x}|}\right)^{\varepsilon-1} e^{-s^{2} / 4 \lambda} d s \geq \frac{1}{|\mathbf{x}|^{1-\varepsilon}} C_{2}
$$

with

$$
C_{2}:=\int_{R_{0}-r_{0}}^{\infty} e^{-s^{2} / 4 \lambda} d s
$$

Hence

$$
J_{1}(\mathbf{x}) \geq \frac{1}{|\mathbf{x}|^{1-\varepsilon}} \min \left\{C_{1}, C_{2}\right\}, \quad|\mathbf{x}| \geq r_{0}
$$


As for $J_{2}(\mathbf{x})$, we have

$$
J_{2}(\mathbf{x})=\frac{1}{|\mathbf{x}|^{1-\varepsilon}} \int_{1+R_{0} /|\mathbf{x}|}^{\infty} \frac{e^{-(r|\mathbf{x}|)^{2} / 4 \lambda}(r|\mathbf{x}|)}{r(r-1)^{1-\varepsilon}} d r .
$$

Hence, for all $r_{1}>0$, we have

$$
J_{2}(\mathbf{x}) \leq \frac{1}{|\mathbf{x}|^{1-\varepsilon}} M_{r_{1}} C_{3}, \quad \forall|\mathbf{x}| \geq r_{1}
$$

with

$$
M_{r_{1}}:=\sup _{s \geq R_{0}+r_{1}}\left(s e^{-s^{2} / 8 \lambda}\right), \quad C_{3}:=\int_{1}^{\infty} \frac{e^{-r^{2} r_{1}^{2} / 8 \lambda}}{r(r-1)^{1-\varepsilon}} d r .
$$

Thus, taking $r_{1} \geq r_{0}$, we obtain

$$
I_{1}(\mathbf{x}) \leq-\frac{a_{1}}{|\mathbf{x}|^{2-\varepsilon}}, \quad \forall|\mathbf{x}| \geq r_{1}
$$

with

$$
a_{1}:=4 \pi \lambda a\left(\min \left\{C_{1}, C_{2}\right\}-M_{r_{1}} C_{3}\right) .
$$

Since $\lim _{r_{1} \rightarrow \infty} M_{r_{1}}=0$, we can take $r_{1}$ such that $a_{1}>0$.

It is easy to see that, for all $\alpha \in \mathbb{R}$

$$
\left|I_{2}(\mathbf{x})\right| \leq \frac{N_{r_{2}}(\alpha)}{|\mathbf{x}|^{\alpha}}, \quad|\mathbf{x}| \geq r_{2}>0
$$

with

$$
N_{r_{2}}(\alpha):=\sup _{|\mathbf{x}| \geq r_{2}}\left(|\mathbf{x}|^{\alpha} e^{-|\mathbf{x}|^{2} / 4 \lambda} e^{|\mathbf{x}| R_{0} / 2 \lambda}\right)\left(\int_{|\mathbf{y}| \leq R_{0}} e^{-\mathbf{y}^{2} / 4 \lambda}|V(\mathbf{y})| d \mathbf{y}\right)<\infty .
$$

Note that $\lim _{r_{2} \rightarrow \infty} N_{r_{2}}(\alpha)=0$. Hence we can take $r_{2}$ such that $a^{\prime}:=a_{1}-N_{r_{2}}(\alpha)>0$ with $\alpha=2-\varepsilon$. Then, taking $R_{0}^{\prime}:=\max \left\{r_{1}, r_{2}\right\}$, we have (3.6).

Lemma 3.8 Assume (1.5). Suppose that there exist constants $b>0$ and $R_{0}>0$ such that

$$
V(\mathbf{x}) \geq-\frac{b}{|\mathbf{x}|^{2}}, \quad \forall|\mathbf{x}| \geq R_{0}
$$

Then, for each $\lambda>0$, there exist constants $b^{\prime}>0$ and $R_{0}^{\prime}>0$ such that

$$
V_{\lambda}(\mathbf{x}) \geq-\frac{b^{\prime}}{|\mathbf{x}|^{2}}, \quad \forall|\mathbf{x}| \geq R_{0}^{\prime}
$$

Proof. We decompose $V_{\lambda}$ as in (3.7) with (3.8). In the same way as in the proof of Lemma 3.7, we can show that, for all $\mathbf{x} \neq \mathbf{0}$,

$$
\begin{aligned}
I_{1}(\mathbf{x}) & \geq-\frac{4 \pi b \lambda}{|\mathbf{x}|} \int_{R_{0}}^{\infty} \frac{e^{-\left(|\mathbf{x}|^{2}+r^{2}\right) / 4 \lambda+|\mathbf{x}| r / 2 \lambda}}{r} d r \\
& =-\frac{4 \pi b \lambda}{|\mathbf{x}|^{2}} \int_{R_{0}-|\mathbf{x}|}^{\infty} \frac{e^{-s^{2} / 4 \lambda}}{\left(1+\frac{s}{|\mathbf{x}|}\right)} d s \\
& \geq-\frac{4 \pi b \lambda}{|\mathbf{x}|^{2}} \int_{-\infty}^{\infty} e^{-s^{2} / 4 \lambda} d s=-\frac{(4 \pi \lambda)^{3 / 2} b}{|\mathbf{x}|^{2}}
\end{aligned}
$$


By (3.9), we have

$$
I_{2}(\mathbf{x}) \geq-\frac{N_{r_{2}}(2)}{|\mathbf{x}|^{2}}, \quad|\mathbf{x}| \geq r_{2}>0
$$

Thus (3.11) follows.

\section{Discrete Spectrum of the Effective Hamiltonian}

As is well known, every $U \in \mathcal{R}+L^{\infty}\left(\mathbb{R}^{3}\right)$ as a multiplication operator on $L^{2}\left(\mathbb{R}^{3}\right)$ is infinitesimally form-bounded with respect to $\Delta$ on $L^{2}\left(\mathbb{R}^{3}\right)$ [11, Theorem X.19]. Hence, by the KLMN theorem [11, Theorem X.17], there exists a unique self-adjoint operator $S_{U}$ such that $D\left(\left|S_{U}\right|^{1 / 2}\right)=D\left((-\Delta)^{1 / 2}\right)$ and

$$
\begin{aligned}
& \int_{\mathbb{R}} \mu d\left\langle f, E_{S_{U}}(\mu) g\right\rangle \\
& =-\frac{\hbar^{2}}{2 m}\left\langle(-\Delta)^{1 / 2} f,(-\Delta)^{1 / 2} g\right\rangle+\int_{\mathbb{R}^{3}} f(\mathbf{x})^{*} U(\mathbf{x}) g(\mathbf{x}) d \mathbf{x}, \quad f, g \in D\left((-\Delta)^{1 / 2}\right) .
\end{aligned}
$$

We write $S_{U}$ as

$$
S_{U}:=-\frac{\hbar^{2}}{2 m} \Delta \dot{+} U
$$

By Lemma 3.4, for $V \in \mathcal{R} \cap L^{1}\left(\mathbb{R}^{3}\right)+L^{\infty}\left(\mathbb{R}^{3}\right)$, we can define a self-adjoint operator $\widetilde{H}_{\lambda}(\lambda \geq 0)$ by

$$
\widetilde{H}_{\lambda}:=-\frac{\hbar^{2}}{2 m} \Delta \dot{+} V_{\lambda}
$$

Remark 4.1 The operator $\widetilde{H}_{\lambda}$ is a self-adjoint extension of $H_{\lambda}$. If $V \in L^{2}\left(\mathbb{R}^{3}\right) \cap L^{1}\left(\mathbb{R}^{3}\right)+$ $L^{\infty}\left(\mathbb{R}^{3}\right)$, then $\widetilde{H}_{\lambda}=H_{\lambda}$, since $H_{\lambda}$ is self-adjoint with $D\left(H_{\lambda}\right)=D(\Delta)$ in this case.

Theorem 4.2 Let $V \in \mathcal{R} \cap L^{1}\left(\mathbb{R}^{3}\right)+L_{\varepsilon}^{\infty}\left(\mathbb{R}^{3}\right)$. Then, for all $\lambda \geq 0$, $\sigma_{\mathrm{ess}}\left(\widetilde{H}_{\lambda}\right)=[0, \infty)$ and $\sigma_{\text {disc }}\left(\widetilde{H}_{\lambda}\right) \subset(-\infty, 0)$. Moreover, the following (i) and (ii) hold:

(i) Suppose that (3.5) holds. Then, for all $\lambda \geq 0, \sigma_{\mathrm{disc}}\left(\widetilde{H}_{\lambda}\right)$ is infinite.

(ii) Suppose that (3.10) holds. Then, for all $\lambda \geq 0, \sigma_{\text {disc }}\left(\widetilde{H}_{\lambda}\right)$ is finite.

Proof. The following fact is well known [12, p.118, Example 7]: If $U \in \mathcal{R}+L_{\varepsilon}^{\infty}\left(\mathbb{R}^{3}\right)$, then $\sigma_{\text {ess }}\left(S_{U}\right)=[0, \infty)$, where $S_{U}$ is defined by $(4.1)$, and hence $\sigma_{\text {disc }}\left(S_{U}\right) \subset(-\infty, 0)$. By Lemma 3.6, we can apply this theorem to $U=V_{\lambda}$. Thus the first half of the present theorem follows.

(i) In this case, we have (3.6). Hence, by a general theorem [11, Theorem XIII.6-(a)], $\sigma_{\text {disc }}\left(\widetilde{H}_{\lambda}\right)$ is infinite for all $\lambda \geq 0$.

(ii) In this case, we have (3.11). Hence a general theorem [11, Theorem XIII.6-(b)] implies that $\sigma_{\text {disc }}\left(\widetilde{H}_{\lambda}\right)$ is finite for all $\lambda \geq 0$.

Theorem 4.2 shows that, for every $V \in \mathcal{R} \cap L^{1}\left(\mathbb{R}^{3}\right)+L_{\varepsilon}^{\infty}\left(\mathbb{R}^{3}\right)$ with condition (3.5) (resp. (3.10)), the infiniteness (resp. finiteness) of the discrete spectrum of $\widetilde{H}_{0}=-\hbar^{2} \Delta / 2 m \dot{+} V$ is maintained under the change of $V$ to $V_{\lambda}$. 


\section{Spherically Symmetric Potentials}

In this section we consider the case where $V$ is in a class of spherically symmetric potentials on $\mathbb{R}^{3}$. Let $V$ be given by the following form:

$$
V(\mathbf{x})=\frac{u(|\mathbf{x}|)}{|\mathbf{x}|}, \quad \mathbf{x} \in \mathbb{R}^{3} \backslash\{\mathbf{0}\}
$$

with $u:[0, \infty) \rightarrow \mathbb{R}$ being bounded and continuously differentiable on $[0, \infty)$ with $u^{\prime}$ - the derivative of $u$-bounded on $[0, \infty)$. Note that $V$ has singularity at $\mathbf{x}=\mathbf{0}$ if $u(\mathbf{0}) \neq$ 0 . Hence this class of $V$ includes classes of potentials different from those discussed in Subsection 2.1.

\subsection{General aspects}

Theorem 5.1 Let $V$ be given by (5.1). Then, for all $\lambda \geq 0, H_{\lambda}$ is self-adjoint with $D\left(H_{\lambda}\right)=D(\Delta)$ and bounded below. Moreover

$$
\sigma_{\text {ess }}\left(H_{\lambda}\right)=[0, \infty), \quad \sigma_{\text {disc }}\left(H_{\lambda}\right) \subset(-\infty, 0)
$$

Proof. For every $\varepsilon>0$, let $R>\|u\|_{\infty} / \varepsilon$ and define

$$
V_{1}(\mathbf{x}):=\frac{u(\mathbf{x}) \chi_{(0, R)}(|\mathbf{x}|)}{|\mathbf{x}|}, \quad V_{2}(\mathbf{x}):=\frac{u(\mathbf{x}) \chi_{[R, \infty)}(|\mathbf{x}|)}{|\mathbf{x}|},
$$

where, for a set $S \subset \mathbb{R}, \chi_{S}$ denotes the characteristic function of $S$. Then it is easy to see that $V_{1} \in L^{2}\left(\mathbb{R}^{3}\right),\left\|V_{2}\right\|_{\infty}<\varepsilon$ and $V=V_{1}+V_{2}$ on $\mathbb{R}^{3} \backslash\{\mathbf{0}\}$. Hence $V \in L^{2}\left(\mathbb{R}^{3}\right)+L_{\varepsilon}^{\infty}\left(\mathbb{R}^{3}\right)$. Therefore, by Theorem 2.10, we obtain the desired results.

We next estimate $\left\|\left(V_{\lambda}-V\right)(H-z)^{-1}\right\|$. We have by direct computations

$$
V_{\lambda}=V+W_{\lambda 1}+W_{\lambda 2}+W_{\lambda 3},
$$

with

$$
\begin{aligned}
& W_{\lambda 1}(\mathbf{x}):=\frac{1}{\sqrt{\pi}|\mathbf{x}|} \int_{-|\mathbf{x}| / 2 \sqrt{\lambda}}^{\infty} e^{-s^{2}}\{u(|\mathbf{x}|+2 \sqrt{\lambda} s)-u(|\mathbf{x}|)\} d s \\
& W_{\lambda 2}(\mathbf{x}):=-\frac{u(|\mathbf{x}|)}{\sqrt{\pi}|\mathbf{x}|} \operatorname{Erfc}(|\mathbf{x}| / 2 \sqrt{\lambda}) \\
& W_{\lambda 3}(\mathbf{x}):=-\frac{1}{\sqrt{\pi}|\mathbf{x}|} \int_{0}^{\infty} e^{-(s+|\mathbf{x}| / 2 \sqrt{\lambda})^{2}} u(2 \sqrt{\lambda} s) d s
\end{aligned}
$$

where

$$
\operatorname{Erfc}(x):=\int_{x}^{\infty} e^{-y^{2}} d y, \quad x \in \mathbb{R},
$$

the Gauss error function. Hence $V_{\lambda}$ also is spherically symmetric. 
Example 5.2 In the case where $V$ is a Coulomb type potential

$$
V^{(\gamma)}(\mathbf{x})=-\frac{\gamma}{|\mathbf{x}|}, \quad \mathbf{x} \in \mathbb{R}^{d} \backslash\{0\}
$$

with $\gamma \in \mathbb{R} \backslash\{0\}$ being a constant, we have

$$
V_{\lambda}(\mathbf{x})=V_{\lambda}^{(\gamma)}(\mathbf{x}):=-\frac{\gamma}{|\mathbf{x}|}+W_{\lambda}^{(\gamma)}(\mathbf{x})
$$

where

$$
W_{\lambda}^{(\gamma)}(\mathbf{x}):=\frac{2 \gamma}{\sqrt{\pi}|\mathbf{x}|} \operatorname{Erfc}(|\mathbf{x}| / 2 \sqrt{\lambda}), \quad r>0
$$

It is easy to see that $V$ is in $L^{2}\left(\mathbb{R}^{3}\right)+L^{\infty}\left(\mathbb{R}^{3}\right)$. Hence it is infinitesimally small with respect to $-\Delta$ (see, e.g., proof of [11, Theorem X.15]): for every $\varepsilon>0$, there exists a constant $b_{\varepsilon}>0$ such that

$$
\|V f\| \leq \varepsilon\left\|-\frac{\hbar^{2}}{2 m} \Delta f\right\|+b_{\varepsilon}\|f\|, \quad f \in D(-\Delta) .
$$

Hence, by the Kato-Rellich theorem [11, Theorem X.12], $H$ is self-adjoint with $D(H)=$ $D(-\Delta)$ and boundd below. By Lemma 2.1, $V_{\lambda}$ also is in $L^{2}\left(\mathbb{R}^{3}\right)+L^{\infty}\left(\mathbb{R}^{3}\right)$ for all $\lambda>0$. Hence $H_{\lambda}$ is self-adjoint with $D\left(H_{\lambda}\right)=D(-\Delta)$ and bounded below.

We note that (5.11) implies that

$$
\left\|-\frac{\hbar^{2}}{2 m} \Delta f\right\| \leq \frac{1}{1-\varepsilon}\|H f\|+\frac{b_{\varepsilon}}{1-\varepsilon}\|f\|, \quad 0<\varepsilon<1 .
$$

A key fact is given by the next lemma:

Lemma 5.3 For all $z \in \rho(H)$ and $p \in(0,1 / 4)$, there exists a constant $C(p, z)>0$ independent of $\lambda>0$ such that

$$
\left\|\left(V_{\lambda}-V\right)(H-z)^{-1}\right\| \leq C(p, z)\left(\sqrt{\lambda}+\lambda^{p}+\lambda^{1 / 4}\right)
$$

and, for all compact sets $S \subset \rho(H) \sup _{z \in S} C(p, z)<\infty$. In particular,

$$
\lim _{\lambda \rightarrow 0}\left\|\left(V_{\lambda}-V\right)(H-z)^{-1}\right\|=0
$$

uniformly in $z$ on each compact set $S \subset \rho(H)$ and, for all $f \in D(\Delta)$ and $p \in(0,1 / 4)$,

$$
\left\|\left(V_{\lambda}-V\right) f\right\|=o\left(\lambda^{p}\right) \quad(\lambda \rightarrow 0) .
$$

Proof. Throughout the proof, $C_{j}(j=1,2, \cdots)$ denotes a positive constant independent of $\lambda$ and $z \in \rho(H)$. To prove (5.13), we need only to prove

$$
\left\|W_{\lambda j}(H-z)^{-1}\right\| \leq C_{j}(p, z)\left(\sqrt{\lambda}+\lambda^{p}+\lambda^{1 / 4}\right), \quad j=1,2,3,
$$

where $C_{j}(p, z)$ is a positive constant independent of $\lambda>0$ and $\sup _{z \in S} C_{j}(p, z)<\infty$ for all compact sets $S \subset \rho(H)$. 
We have

$$
u(|\mathbf{x}|+2 \sqrt{\lambda} s)-u(|\mathbf{x}|)=\int_{|\mathbf{x}|}^{|\mathbf{x}|+2 \sqrt{\lambda} s} u^{\prime}(r) d r .
$$

Hence

$$
|u(|\mathbf{x}|+2 \sqrt{\lambda} s)-u(|\mathbf{x}|)| \leq 2 \sqrt{\lambda}|s|\left\|u^{\prime}\right\|_{\infty} .
$$

By this estimate and (5.4), we obtain

$$
\left|W_{\lambda 1}(\mathbf{x})\right| \leq \frac{C_{1}}{|\mathbf{x}|} \sqrt{\lambda}
$$

with a constant $C_{1}$. Since $1 /|\mathbf{x}|$ is in $L^{2}\left(\mathbb{R}^{3}\right)+L^{\infty}\left(\mathbb{R}^{3}\right)$, an estimate like (5.11) holds. Namely, for every $\varepsilon_{1}>0$, there exists a constant $c_{\varepsilon_{1}}>0$ such that

$$
\left\||\mathbf{x}|^{-1} f\right\| \leq \varepsilon_{1}\left\|-\frac{\hbar^{2}}{2 m} \Delta f\right\|+c_{\varepsilon_{1}}\|f\|, \quad f \in D(-\Delta) .
$$

By (5.12), we have

$$
\left\||\mathbf{x}|^{-1} f\right\| \leq \frac{\varepsilon_{1}}{1-\varepsilon}\|H f\|+d_{\varepsilon, \varepsilon_{1}}\|f\| \quad(0<\varepsilon<1)
$$

with a constant $d_{\varepsilon, \varepsilon_{1}}>0$. Hence we obtain for all $z \in \rho(H)$

$$
\left\|W_{\lambda 1}(H-z)^{-1}\right\| \leq \sqrt{\lambda} C_{2}\left(\left\|H(H-z)^{-1}\right\|+\left\|(H-z)^{-1}\right\|\right)
$$

with a constant $C_{2}>0$. Therefore, taking

$$
C_{1}(p, z)=C_{2}\left(\left\|H(H-z)^{-1}\right\|+\left\|(H-z)^{-1}\right\|\right)
$$

we have (5.16) with $j=1$. Let $S \subset \rho(H)$ be a compact set. Then it is easy to see that

$$
\sup _{z \in S}\left(\left\|H(H-z)^{-1}\right\|+\left\|(H-z)^{-1}\right\|\right)<\infty .
$$

Hence $\sup _{z \in S} C_{1}(z \cdot p)<\infty$.

Let $f \in D(\Delta)$ and $0<p<1 / 4$. Then we have

$$
\left\|W_{\lambda 2} f\right\|^{2}=I_{1}(f)+I_{2}(f)
$$

with

$$
I_{1}(f):=\int_{|\mathbf{x}| \leq \lambda^{2 p}}\left|W_{\lambda 2}(\mathbf{x})\right|^{2}|f(\mathbf{x})|^{2} d \mathbf{x}, \quad I_{2}(f):=\int_{|\mathbf{x}|>\lambda^{2 p}}\left|W_{\lambda 2}(\mathbf{x})\right|^{2}|f(\mathbf{x})|^{2} d \mathbf{x} .
$$

Since $\operatorname{Erfc}(0)=\sqrt{\pi} / 2$, we have

$$
\begin{aligned}
I_{1}(f) & \leq\left(\frac{\sqrt{\pi}}{2}\right)^{2} \frac{1}{\pi}\|u\|_{\infty}^{2}\|f\|_{\infty}^{2} \int_{|\mathbf{x}| \leq \lambda^{2 p}} \frac{1}{|\mathbf{x}|^{2}} d \mathbf{x} \\
& =C_{4} \lambda^{2 p}\|f\|_{\infty}^{2}
\end{aligned}
$$


with a constant $C_{4}$ independent of $\lambda$. Recall that, for every $a>0$, there exists a constant $b$ such that

$$
\|f\|_{\infty} \leq a\|\Delta f\|+b\|f\|
$$

(see, e.g., [11, (X.23)]). By this estimate and (5.12), we obtain

$$
I_{1}(f) \leq C_{5} \lambda^{2 p}\left(\|H f\|^{2}+\|f\|^{2}\right)
$$

with a constant $C_{5}$.

As for $I_{2}(f)$, we have

$$
I_{2}(f) \leq C_{6} \operatorname{Erfc}\left(\lambda^{(4 p-1) / 2} / 2\right)^{2}\left\||\mathbf{x}|^{-1} f\right\|^{2} \leq C_{7} \operatorname{Erfc}\left(\lambda^{(4 p-1) / 2} / 2\right)^{2}\left(\|H f\|^{2}+\|f\|^{2}\right)
$$

with constants $C_{6}$ and $C_{7}$, where we have used (5.17). Thus we obtain

$$
\left\|W_{\lambda 2}(H-z)^{-1}\right\| \leq C_{8} \sqrt{\lambda^{2 p}+\operatorname{Erfc}\left(\lambda^{(4 p-1) / 2} / 2\right)^{2}}\left(\left\|H(H-z)^{-1}\right\|+\left\|(H-z)^{-1}\right\|\right)
$$

with a constant $C_{8}$. We have for all $\eta \in(0,1)$ and $\theta>0$

$$
\begin{aligned}
\operatorname{Erfc}\left(\lambda^{(4 p-1) / 2} / 2\right) & =\int_{1 / 2 \lambda^{(1-4 p) / 2}}^{\infty} e^{-s^{2}} d s \leq e^{-\eta / 4 \lambda^{1-4 p}} \int_{0}^{\infty} e^{-(1-\eta) s^{2}} d s \\
& \leq C_{9}(\eta, \theta) \lambda^{\theta(1-4 p)}
\end{aligned}
$$

with a constant $C_{9}(\eta, \theta)>0$. Hence

$$
\left\|W_{\lambda 2}(H-z)^{-1}\right\| \leq C_{10}(p, \theta)\left(\lambda^{p}+\lambda^{\theta(1-4 p)}\right)\left(\left\|H(H-z)^{-1}\right\|+\left\|(H-z)^{-1}\right\|\right)
$$

with a constant $C_{10}(p, \theta)$. Taking $\theta=p /(1-4 p)$, we obtain (5.16) with $j=2$.

We have

$$
\left|W_{\lambda 3}(\mathbf{x})\right| \leq C_{11} \frac{e^{-|\mathbf{x}|^{2} / 4 \lambda}}{|\mathbf{x}|}
$$

with a constant $C_{11}$. Hence we have for all $f \in D(\Delta)$

$$
\left\|W_{\lambda 3} f\right\|^{2} \leq C_{11}^{2}\left(K_{1}+K_{2}\right)
$$

with

$$
K_{1}:=\int_{|\mathbf{x}| \leq R} \frac{e^{-|\mathbf{x}|^{2} / 2 \lambda}}{|\mathbf{x}|^{2}}|f(\mathbf{x})|^{2} d \mathbf{x}, \quad K_{2}:=\int_{|\mathbf{x}|>R} \frac{e^{-|\mathbf{x}|^{2} / 2 \lambda}}{|\mathbf{x}|^{2}}|f(\mathbf{x})|^{2} d \mathbf{x}
$$

where $R>0$ is a constant. It is easy to see that

$$
K_{1} \leq C_{12} \sqrt{\lambda}\|f\|_{\infty}^{2}, \quad K_{2} \leq \frac{e^{-R^{2} / 2 \lambda}}{R^{2}}\|f\|^{2}
$$

with a constant $C_{12}$. Hence, as in the preceding cases, we have

$$
\left\|W_{\lambda 3}(H-z)^{-1}\right\| \leq C_{13}\left(\lambda^{1 / 4}+e^{-R^{2} / 4 \lambda}\right)\left(\left\|H(H-z)^{-1}\right\|+\left\|(H-z)^{-1}\right\|\right)
$$

with a constant $C_{13}$. Thus (5.16) with $j=3$ holds. 
Estimate (5.13) obviously implies the uniform convergence (5.14) on each compact set $S \subset \rho(H)$.

Let $f \in D(\Delta)$ and $p \in(0,1 / 4)$. Then there exists a constant $\varepsilon>0$ such that $0<p+\varepsilon<1 / 4$. For all $\lambda \in(0,1)$, we have $\sqrt{\lambda}, \lambda^{1 / 4}<\lambda^{p+\varepsilon}$. Hence, we have for all $\lambda \in(0,1)$

$$
\left\|\left(V_{\lambda}-V\right) f\right\| \leq\left\|\left(V_{\lambda}-V\right)(H-i)^{-1}\right\|\|(H-i) f\| \leq 3 C(p+\varepsilon, i)\|(H-i) f\| \lambda^{p+\varepsilon},
$$

which implies (5.15).

By Lemma 5.3, we can apply a general perturbation theory given in Appendix in the present paper to investigate the discrete spectrum of $H_{\lambda}$.

Suppose that $H$ has an isolated eigenvalue $E_{0} \in \mathbb{R}$ with finite multiplicity $m\left(E_{0}\right)$ $\left(1 \leq m\left(E_{0}\right)<\infty\right)$. Let $r$ be a constant satisfying

$$
0<r<\min _{E \in \sigma(H) \backslash\left\{E_{0}\right\}}\left|E-E_{0}\right|
$$

and

$$
C_{r}\left(E_{0}\right):=\left\{z \in \mathbb{C}|| z-E_{0} \mid=r\right\},
$$

which is a subset of $\rho(H)$. Let

$$
n_{r}:=r \sup _{z \in C_{r}\left(E_{0}\right)}\left\|(H-z)^{-1}\right\|, \quad r_{\lambda}:=\sup _{z \in C_{r}\left(E_{0}\right)}\left\|\left(V_{\lambda}-V\right)(H-z)^{-1}\right\| .
$$

The next theorem follows from a simple application of Theorem A.3 with $A=H$ and $B_{\lambda}=V_{\lambda}-V$ :

Theorem 5.4 Let $\lambda>0$ and $r_{\lambda}<1 /\left(1+n_{r}\right)$. Then, $H_{\lambda}$ has exactly $m\left(E_{0}\right)$ eigenvalues in the interval $\left(E_{0}-r, E_{0}+r\right)$, counting multiplicities, and $\sigma\left(H_{\lambda}\right) \cap\left(E_{0}-r, E_{0}+r\right)$ consists of only these eigenvalues.

In the case where $E_{0}$ is a simple eigenvalue of $H$, one can obtain more detailed results:

Corollary 5.5 Let $\lambda>0$ and $r_{\lambda}<1 /\left(1+n_{r}\right)$. Suppose that $m\left(E_{0}\right)=1$ and $\Omega_{0}$ is a normalized eigenvector of $H$ with eigenvalue $E_{0}$. Then, $H_{\lambda}$ has exactly one simple eigenvalue $E_{\lambda}$ in the interval $\left(E_{0}-r, E_{0}+r\right)$ with formula

$$
E_{\lambda}=E_{0}+\frac{\left\langle\Omega_{0},\left(V_{\lambda}-V\right) \Omega_{0}\right\rangle+S_{n}(\lambda)}{1+\sum_{n=1}^{\infty} T_{n}(\lambda)},
$$

where

$$
\begin{aligned}
& S_{n}(\lambda):=\frac{(-1)^{n+1}}{2 \pi i} \int_{C_{r}\left(E_{0}\right)} d z\left\langle\Omega_{0},\left[\left(V_{\lambda}-V\right)(H-z)^{-1}\right]^{n+1} \Omega_{0}\right\rangle, \\
& T_{n}(\lambda):=\frac{(-1)^{n+1}}{2 \pi i} \int_{C_{r}\left(E_{0}\right)} d z \frac{\left\langle\Omega_{0},\left[\left(V_{\lambda}-V\right)(H-z)^{-1}\right]^{n} \Omega_{0}\right\rangle}{E_{0}-z},
\end{aligned}
$$


and $\sigma\left(H_{\lambda}\right) \cap\left(E_{0}-r, E_{0}+r\right)=\left\{E_{\lambda}\right\}$. Moreover, a normalized eigenvector of $H_{\lambda}$ with eigenvalue $E_{\lambda}$ is given by

$$
\Omega_{\lambda}=\frac{\Omega_{0}+\sum_{n=1}^{\infty} \Omega_{\lambda, n}}{\sqrt{1+\sum_{n=1}^{\infty} T_{n}(\lambda)}}
$$

where

$$
\Omega_{\lambda, n}:=\frac{(-1)^{n+1}}{2 \pi i} \int_{C_{r}\left(E_{0}\right)} d z(H-z)^{-1}\left[\left(V_{\lambda}-V\right)(H-z)^{-1}\right]^{n} \Omega_{0} .
$$

Proof. This is a simple application of Corollary A.4 to the case where $A=H$ and $B_{\lambda}=V_{\lambda}-V$.

\subsection{Reductions of $H_{\lambda}$ to closed subspaces}

As is well known (e.g., [11, p.160, Example 4]), the Hilbert space $L^{2}\left(\mathbb{R}^{3}\right)$ has the orthogonal decomposition

$$
L^{2}\left(\mathbb{R}^{3}\right)=\oplus_{\ell=0}^{\infty} \oplus_{s=-\ell}^{\ell} \mathcal{H}_{\ell}^{s}
$$

with

$$
\mathcal{H}_{\ell}^{s}=L^{2}\left([0, \infty), r^{2} d r\right) \otimes\left\{\alpha Y_{\ell}^{s} \mid \alpha \in \mathbb{C}\right\},
$$

where $Y_{\ell}^{s}$ is the spherical harmonics with index $(\ell, s)$ :

$$
\begin{aligned}
Y_{\ell}^{s}(\theta, \phi):=(-1)^{s} \sqrt{\frac{(\ell-s) !}{(\ell+s) !} \sqrt{\frac{2 \ell+1}{4 \pi}}} P_{\ell}^{s}(\cos \theta) e^{i s \phi} \\
\quad \theta \in[0, \pi], \phi \in[0,2 \pi), s=-\ell, \ell+1, \cdots, 0, \cdots, \ell-1, \ell
\end{aligned}
$$

with $P_{\ell}^{s}$ being the associated Legendre function:

$$
P_{\ell}^{s}(x):=\left(1-x^{2}\right)^{s / 2} \frac{d^{s}}{d x^{s}} \frac{(-1)^{\ell}}{2^{\ell} \ell !}\left(\frac{d}{d x}\right)^{\ell}\left(1-x^{2}\right)^{\ell}, \quad|x|<1 .
$$

We have

$$
\int_{0}^{\pi} d \theta \int_{0}^{2 \pi} d \phi \sin \theta Y_{\ell}^{s}(\theta, \phi)^{*} Y_{\ell^{\prime}}^{s^{\prime}}(\theta, \phi)=\delta_{\ell \ell^{\prime}} \delta_{s s^{\prime}}
$$

where $\delta_{a b}$ denotes the Kronecker delta.

Since $V_{\lambda}(\lambda \geq 0)$ under consideration is spherically symmetric, $H_{\lambda}$ is reduced by each $\mathcal{H}_{\ell}^{s}\left[11\right.$, p.160, Example 4]. We denote the reduced part of $H_{\lambda}$ by $H_{\lambda}^{\ell, s}$. Explicitly it is of the form

$$
\begin{aligned}
\left(H_{\lambda}^{\ell, s} f \otimes Y_{\ell}^{s}\right)(r, \phi, \theta)= & \left(-\frac{\hbar^{2}}{2 m} \frac{d^{2}}{d r^{2}}+\widetilde{V}_{\lambda}(r)-\frac{\hbar^{2}}{2 m} \frac{2}{r} \frac{d}{d r}\right) f(r) Y_{\ell}^{s}(\theta, \phi) \\
& +\frac{\ell(\ell+1)}{r^{2}} f(r) Y_{\ell}^{s}(\theta, \phi), \quad f \in C_{0}^{\infty}(0, \infty)
\end{aligned}
$$

where $\widetilde{V}_{\lambda}(r):=\left.V_{\lambda}(\mathbf{x})\right|_{r=|\mathbf{x}|}$.

The following result immediately follows:

Corollary 5.6 For each pair $(\ell, s)(\ell \in\{0\} \cup \mathbb{N}, s=-\ell,-\ell+1, \cdots, \ell)$, Theorem 5.4 and Corollary 5.5 with $H_{\lambda}$ replaced by $H_{\lambda}^{\ell, s}$ hold. 


\section{Energy Level Shifts in a Hydrogen-like Atom}

In this section we apply the results established in the preceding section to a hydrogen-like atom and derive formulas for the energy level shifts due to the interaction of the electron with the quantum radiation field, including the Lamb shift. Thus we consider the case where $V$ is a Coulomb type potential $V^{(\gamma)}(\gamma>0)$ defined by (5.8). Hence, in this case, the unperturbed Hamiltonian is given by

$$
H(\gamma):=-\frac{\hbar^{2}}{2 m} \Delta+V^{(\gamma)}
$$

We remark that the Hamiltonian of a hydrogen-like atom is given by $H(\gamma)$ with $\gamma=$ $Z e^{2} / 4 \pi(Z \in \mathbb{N}, e$ is the fundamental charge; we use the rationalized CGS Gauss unit system where the dielectric constant in the vacuum is equal to 1$), q=-e$ and $m$ being the electron mass.

It is well known that

$$
\sigma_{\text {disc }}(H(\gamma))=\left\{E_{n} \mid n \in \mathbb{N}\right\}, \quad \sigma_{\text {ess }}(H(\gamma))=[0, \infty)
$$

with

$$
E_{n}=-\frac{1}{2} \frac{m \gamma^{2}}{n^{2} \hbar^{2}}
$$

The multiplicity of $E_{n}$ is $n^{2}$ in such a way that $E_{n}$ is a unique simple eigenvalue of the reduced part $H^{\ell, s}(\gamma)$ of $H(\gamma)(0 \leq \ell \leq n-1)$ to the closed subspace $\mathcal{H}_{\ell}^{s}$ with a normalized eigenfunction

$$
\psi_{n, \ell, s}(\mathbf{x}):=C_{n, \ell} e^{-\beta_{n} r / 2}\left(\beta_{n} r\right)^{\ell} L_{n+\ell}^{2 \ell+1}\left(\beta_{n} r\right) Y_{\ell}^{s}(\theta, \phi), \quad r=|\mathbf{x}|, \ell=0,1, \cdots, n-1,
$$

where

$$
\beta:=\frac{2 m \gamma}{\hbar^{2} n},
$$

$L_{n}^{k}(0 \leq k \leq n)$ is the Laguerre associated polynomial with order $n-k$, i.e.,

$$
L_{n}^{k}(x)=\frac{d^{k}}{d x^{k}} L_{n}(x), \quad x \in \mathbb{R}
$$

with $L_{n}(x)$ being the $n$-th Laguerre polynomial (we follow the notation in $[8, \S 2.17]$ ), and

$$
C_{n, \ell}:=\frac{\beta_{n}^{3 / 2} \sqrt{(n-\ell-1) !}}{\sqrt{[(n+\ell) !]^{32 n}}} .
$$

Let

$$
H_{\lambda}(\gamma):=-\frac{\hbar^{2}}{2 m} \Delta+V_{\lambda}^{(\gamma)}, \quad \lambda>0
$$

where $V_{\lambda}^{(\gamma)}$ is defined by (5.9). It follows that

$$
H_{\lambda}(\gamma)=H(\gamma)+W_{\lambda}^{(\gamma)}
$$

where $W_{\lambda}^{(\gamma)}$ is given by (5.10). The following theorem is a nonperturbative result on the discrete spectrum and the essential spectrum of $H_{\lambda}(\gamma)$ : 
Theorem 6.1 For all $\lambda>0$ and $\gamma>0, H_{\lambda}(\gamma)$ is self-adjoint with $D\left(H_{\lambda}(\gamma)\right)=D(\Delta)$ and bounded below. Moreover, $\sigma_{\text {disc }}\left(H_{\lambda}(\gamma)\right)$ is infinite and

$$
\sigma_{\text {disc }}\left(H_{\lambda}(\gamma)\right) \subset(-\infty, 0), \quad \sigma_{\text {ess }}\left(H_{\lambda}(\gamma)\right)=[0, \infty) .
$$

Proof. We can apply Theorem 5.1 to the case $u(\mathbf{x})=-\gamma$ to obtain the stated results except the infiniteness of $\sigma_{\text {disc }}\left(H_{\lambda}(\gamma)\right)$.

One can easily show that $V^{(\gamma)}$ is in $\mathcal{R} \cap L^{1}\left(\mathbb{R}^{3}\right)+L_{\varepsilon}^{\infty}\left(\mathbb{R}^{3}\right)$ and that $V=V^{(\gamma)}$ satisfies (3.5) with $\varepsilon=1, a=\gamma$. Hence, by Theorem 4.2-(i), $\sigma_{\text {disc }}\left(H_{\lambda}(\gamma)\right)$ is inifinite.

We next consider $H_{\lambda}(\gamma)$ perturbatively. Noting that

$$
\left|E_{n+1}-E_{n}\right|<\left|E_{n}-E_{n-1}\right|, \quad n \geq 2,
$$

we take $r_{n}>0$ such that

$$
r_{n}<\left|E_{n+1}-E_{n}\right|
$$

and set

$$
C_{r_{n}}\left(E_{n}\right):=\left\{z \in \mathbb{C}|| z-E_{n} \mid=r_{n}\right\}
$$

Let

$$
\left.M_{n}:=r_{n} \sup _{z \in C_{r_{n}}\left(E_{n}\right)} \| H(\gamma)-z\right)^{-1}\left\|, \quad R_{\lambda, n}:=\sup _{z \in C_{r_{n}}\left(E_{n}\right)}\right\| W_{\lambda}^{(\gamma)}(H(\gamma)-z)^{-1} \| .
$$

Lemma 6.2 Let $R_{\lambda, n}<1$. Then, $C_{r_{n}}\left(E_{n}\right) \subset \rho\left(H_{\lambda}(\gamma)\right)$ and

$$
\left(H_{\lambda}(\gamma)-z\right)^{-1}=\sum_{p=0}^{\infty}(-1)^{p}(H(\gamma)-z)^{-1}\left[W_{\lambda}^{(\gamma)}(H(\gamma)-z)^{-1}\right]^{p}
$$

in operator norm, uniformly in $z \in C_{r_{n}}\left(E_{n}\right)$.

Proof. We have by (6.3)

$$
H_{\lambda}(\gamma)-z=\left(1+W_{\lambda}^{(\gamma)}(H(\gamma)-z)^{-1}\right)(H(\gamma)-z), \quad z \in \rho(H(\gamma)) .
$$

Then a simple application of the Neumann's expansion gives the desired result.

We denote by $H_{\lambda}^{\ell, s}(\gamma)$ the reduced part of $H_{\lambda}(\gamma)$ to $\mathcal{H}_{\ell}^{s}$.

Theorem 6.3 Let $n \in \mathbb{N}, \ell=0,1, \cdots, n-1$ and $s=-\ell,-\ell+1, \cdots, \ell$. Suppose that $\lambda>0$ and $R_{\lambda, n}<1 /\left(1+M_{n}\right)$. Then, $H_{\lambda}^{\ell, s}(\gamma)$ has a unique simple eigenvalue $E_{n, \ell, s}(\lambda)$ near $E_{n}$ with

$$
E_{n, \ell, s}(\lambda)=E_{n}+\frac{\left\langle\psi_{n, \ell, s}, W_{\lambda}^{(\gamma)} \psi_{n, \ell, s}\right\rangle+\sum_{p=1}^{\infty} F_{n, \ell, s}^{(p)}(\lambda)}{1+\sum_{p=1}^{\infty} G_{n, \ell, s}^{(p)}(\lambda)},
$$

where

$$
\begin{aligned}
& F_{n, \ell, s}^{(p)}(\lambda):=\frac{(-1)^{p+1}}{2 \pi i} \int_{C_{r_{n}}\left(E_{n}\right)}\left\langle\psi_{n, \ell, s},\left[W_{\lambda}^{(\gamma)}(H(\gamma)-z)^{-1}\right]^{p+1} \psi_{n, \ell, s}\right\rangle d z \\
& G_{n, \ell, s}^{(p)}(\lambda):=\frac{(-1)^{p+1}}{2 \pi i} \int_{C_{r_{n}}\left(E_{n}\right)} \frac{\left\langle\psi_{n, \ell, s},\left[W_{\lambda}^{(\gamma)}(H(\gamma)-z)^{-1}\right]^{p} \psi_{n, \ell, s}\right\rangle}{E_{n}-z} d z
\end{aligned}
$$


Moreover, a normalized eigenvector $\psi_{n, \ell, s}^{(\lambda)}$ of $H_{\lambda}^{\ell, s}(\gamma)$ with eigenvalue $E_{n, \ell, s}(\lambda)$ is given by

$$
\psi_{n, \ell, s}^{(\lambda)}=\frac{\psi_{n, \ell, s}+\sum_{p=1}^{\infty} S_{n, \ell, s}^{(p)}(\lambda)}{\sqrt{1+\sum_{p=1}^{\infty} G_{n, \ell, s}^{(p)}(\lambda)}}
$$

where

$$
S_{n, \ell, s}^{(p)}(\lambda):=\frac{(-1)^{p+1}}{2 \pi i} \int_{C_{r_{n}}\left(E_{n}\right)}(H(\gamma)-z)^{-1}\left[W_{\lambda}^{(\gamma)}(H(\gamma)-z)^{-1}\right]^{p} \psi_{n, \ell, s} d z .
$$

Proof. The operator $H^{\ell, s}(\gamma)$ has a unique simple eigenvalue $E_{n}$. Hence Corollary 5.6 yields the desired results.

Let $n \in \mathbb{N}, \lambda>0$ and $R_{\lambda, n}<1 /\left(1+M_{n}\right)$. Then, by Theorem 6.3 , one can define

$$
\Delta E_{n}\left(\ell, s ; \ell^{\prime}, s^{\prime}\right):=E_{n, \ell, s}(\lambda)-E_{n, \ell^{\prime}, s^{\prime}}(\lambda)
$$

for $\ell, \ell^{\prime}=0,1, \cdots, n-1, s, s^{\prime}=-\ell,-\ell+1, \cdots, \ell$ with $(\ell, s) \neq\left(\ell^{\prime}, s^{\prime}\right)$. We call it an energy level shift of $H_{\lambda}(\gamma)$ with respect to the $n$-th energy level.

To compute the energy level shifts of $H_{\lambda}(\gamma)$ approximately in the lowest order in $\lambda>0$ sufficiently small, we need an asymptotic expansion of $E_{n, \ell, s}(\lambda)$ in $\lambda$ as $\lambda \rightarrow 0$. In this respect we have the following theorem:

Theorem 6.4 Under the assumption of Theorem 6.3, the following holds:

$$
E_{n, \ell, s}(\lambda)=E_{n}+4 \pi \gamma\left|\psi_{n, \ell, s}(\mathbf{0})\right|^{2} \lambda+o(\lambda) \quad(\lambda \rightarrow 0) .
$$

To prove this theorem, we need a series of lemmas. We denote by $C\left(\mathbb{R}^{3}\right)$ the set of continuous functions on $\mathbb{R}^{3}$.

Lemma 6.5 Let $f \in L^{2}\left(\mathbb{R}^{3}\right) \cap L^{\infty}\left(\mathbb{R}^{3}\right) \cap C\left(\mathbb{R}^{3}\right)$. Then,

$$
\lim _{\lambda \rightarrow 0} \frac{\left\langle f, W_{\lambda}^{(\gamma)} f\right\rangle}{\lambda}=4 \pi \gamma|f(\mathbf{0})|^{2} .
$$

Proof. We have

$$
\left\langle f, W_{\lambda}^{(\gamma)} f\right\rangle=\frac{2 \gamma}{\sqrt{\pi}}(2 \sqrt{\lambda})^{2} \int_{\mathbb{R}^{3}} \frac{|f(2 \sqrt{\lambda} \mathbf{y})|^{2}}{|\mathbf{y}|} \operatorname{Erfc}(|\mathbf{y}|) d \mathbf{y}
$$

We have

$$
\frac{|f(2 \sqrt{\lambda} \mathbf{y})|^{2}}{|\mathbf{y}|} \operatorname{Erfc}(|\mathbf{y}|) \leq\|f\|_{\infty}^{2} \frac{\operatorname{Erfc}(|\mathbf{y}|)}{|\mathbf{y}|}
$$

and the function $\operatorname{Erfc}(|\mathbf{y}|) /|\mathbf{y}|$ is integrable on $\mathbb{R}^{3}$ with

$$
\int_{\mathbb{R}^{3}} \frac{\operatorname{Erfc}(|\mathbf{y}|)}{|\mathbf{y}|} d \mathbf{y}=\frac{\pi \sqrt{\pi}}{2} .
$$

Hence, by the Lebesgue dominated convergence theorem, we obtain (6.14). 
Lemma 6.6 Let $f \in L^{2}\left(\mathbb{R}^{3}\right) \cap L^{\infty}\left(\mathbb{R}^{3}\right) \cap C\left(\mathbb{R}^{3}\right)$. Then,

$$
\lim _{\lambda \rightarrow 0} \frac{\left\|W_{\lambda}^{(\gamma)} f\right\|^{2}}{\sqrt{\lambda}}=\frac{8 \gamma^{2}}{\pi}|f(\mathbf{0})|^{2} \int_{\mathbb{R}^{3}} \frac{\operatorname{Erfc}(|\mathbf{x}|)^{2}}{|\mathbf{x}|^{2}} d \mathbf{x} .
$$

Proof. Similar to the proof of Lemma 6.5.

Lemma 6.7 Let $f, g \in L^{2}\left(\mathbb{R}^{3}\right) \cap L^{\infty}\left(\mathbb{R}^{3}\right) \cap C\left(\mathbb{R}^{3}\right)$. Then, for all $p \in \mathbb{N}$,

$$
\left\langle f,\left[W_{\lambda}^{(\gamma)}(H(\gamma)-z)^{-1}\right]^{p} W_{\lambda}^{(\gamma)} g\right\rangle=O\left(\lambda^{(p+2) / 2}\right) \quad(\lambda \rightarrow 0)
$$

uniformly in $z \in C_{r_{n}}\left(E_{n}\right)$.

Proof. We first prove (6.16) with $p=1$. Let

$$
K_{0}:=-\frac{\hbar^{2}}{2 m} \Delta,
$$

so that

$$
H(\gamma)=K_{0}+V^{(\gamma)}
$$

Then, for each $z \in C_{r_{n}}\left(E_{n}\right)$ and all $N \in \mathbb{N}$, we have

$$
\begin{aligned}
(H(\gamma)-z)^{-1}= & \sum_{n=0}^{N}(-1)^{n}\left(K_{0}-z\right)^{-1}\left[V^{(\gamma)}\left(K_{0}-z\right)^{-1}\right]^{n} \\
& +(-1)^{N+1}\left[\left(K_{0}-z\right)^{-1} V^{(\gamma)}\right]^{N+1}(H(\gamma)-z)^{-1} .
\end{aligned}
$$

Hence, taking $N=2$, we have

$$
\begin{aligned}
& \left\langle f, W_{\lambda}^{(\gamma)}(H(\gamma)-z)^{-1} W_{\lambda}^{(\gamma)} g\right\rangle \\
= & \left\langle f, W_{\lambda}^{(\gamma)}\left(K_{0}-z\right)^{-1} W_{\lambda}^{(\gamma)} g\right\rangle \\
& +(-1)\left\langle f, W_{\lambda}^{(\gamma)}\left(K_{0}-z\right)^{-1} V^{(\gamma)}\left(K_{0}-z\right)^{-1} W_{\lambda}^{(\gamma)} g\right\rangle \\
& +(-1)^{2}\left\langle f, W_{\lambda}^{(\gamma)}\left(K_{0}-z\right)^{-1}\left[V^{(\gamma)}\left(K_{0}-z\right)^{-1}\right]^{2} W_{\lambda}^{(\gamma)} g\right\rangle \\
& +(-1)^{3}\left\langle f, W_{\lambda}^{(\gamma)}\left[\left(K_{0}-z\right)^{-1} V^{(\gamma)}\right]^{3}(H(\gamma)-z)^{-1} W_{\lambda}^{(\gamma)} g\right\rangle .
\end{aligned}
$$

As is well known, the resolvent $\left(K_{0}-z\right)^{-1}$ of $K_{0}$ at $z \in \rho\left(K_{0}\right)=\mathbb{C} \backslash[0, \infty)$ is an integral operator with integral kernel

$$
L(\mathbf{x}, \mathbf{y}):=\frac{m}{2 \pi \hbar^{2}} \frac{e^{-\sqrt{2 m} \sqrt{-z}|\mathbf{x}-\mathbf{y}| / \hbar}}{|\mathbf{x}-\mathbf{y}|}, \quad \mathbf{x}, \mathbf{y} \in \mathbb{R}^{3}, \mathbf{x} \neq \mathbf{y},
$$

where we take $\sqrt{-z}$ such that $\operatorname{Re} \sqrt{-z}>0$ (e.g., [11, p.59]). Hence $W_{\lambda}^{(\gamma)}\left(K_{0}-z\right)^{-1} W_{\lambda}^{(\gamma)}$ is an integral operator with integral kernel $W_{\lambda}^{(\gamma)}(\mathbf{x}) L(\mathbf{x}, \mathbf{y}) W_{\lambda}^{(\gamma)}(\mathbf{y})$. Using this fact and a change of variable, we have

$$
\left\langle f, W_{\lambda}^{(\gamma)}\left(K_{0}-z\right)^{-1} W_{\lambda}^{(\gamma)} g\right\rangle=C \lambda^{3 / 2} I_{2}(\lambda)
$$


where

$$
\begin{aligned}
& C:=\left(\frac{2 \gamma}{\sqrt{\pi}}\right)^{2}\left(\frac{m}{2 \pi \hbar^{2}}\right) 2^{3} \\
& I_{2}(\lambda):=\int_{\mathbb{R}^{3} \times \mathbb{R}^{3}} f(2 \sqrt{\lambda} \mathbf{x})^{*} g(2 \sqrt{\lambda} \mathbf{y}) \frac{\operatorname{Erfc}(|\mathbf{x}|) \operatorname{Erfc}(|\mathbf{y}|) e^{-2 \sqrt{2 m} \sqrt{-z} \sqrt{\lambda}|\mathbf{x}-\mathbf{y}| / \hbar}}{|\mathbf{x}||\mathbf{x}-\mathbf{y}||\mathbf{y}|} d \mathbf{x} d \mathbf{y} .
\end{aligned}
$$

In the same way as in the proof of Lemma 6.5, we can show that

$$
\lim _{\lambda \rightarrow 0} I_{2}(\lambda)=f(\mathbf{0})^{*} g(\mathbf{0}) \int_{\mathbb{R}^{3} \times \mathbb{R}^{3}} \frac{\operatorname{Erfc}(|\mathbf{x}|) \operatorname{Erfc}(|\mathbf{y}|)}{|\mathbf{x}||\mathbf{x}-\mathbf{y}||\mathbf{y}|} d \mathbf{x} d \mathbf{y},
$$

where the integral on the right hand side is finite. Hence

$$
\left\langle f, W_{\lambda}^{(\gamma)}\left(K_{0}-z\right)^{-1} W_{\lambda}^{(\gamma)} g\right\rangle=O\left(\lambda^{3 / 2}\right) \quad(\lambda \rightarrow 0)
$$

uniformly in $z \in \mathbb{R} \backslash[0, \infty)$. Similarly we can show that, for all $n \geq 1$,

$$
(-1)^{n}\left\langle f, W_{\lambda}^{(\gamma)}\left(K_{0}-z\right)^{-1}\left[V^{(\gamma)}\left(K_{0}-z\right)^{-1}\right]^{n} W_{\lambda}^{(\gamma)} g\right\rangle=O\left(\lambda^{(3+n) / 2}\right) \quad(\lambda \rightarrow 0)
$$

uniformly in $z \in \mathbb{R} \backslash[0, \infty)$.

As for the fourth term on the right hand side of (6.18), we have

$$
\begin{aligned}
& \left|(-1)^{3}\left\langle f, W_{\lambda}^{(\gamma)}\left[\left(K_{0}-z\right)^{-1} V^{(\gamma)}\right]^{3}(H(\gamma)-z)^{-1} W_{\lambda}^{(\gamma)} g\right\rangle\right| \\
& \leq\left\|\left(W_{\lambda}^{(\gamma)}\left[\left(K_{0}-z\right)^{-1} V^{(\gamma)}\right]^{3}\right)^{*} f\right\|\left\|(H(\gamma)-z)^{-1} W_{\lambda}^{(\gamma)} g\right\| \\
& \leq \frac{M_{n}}{r_{n}}\left\|\left[V^{(\gamma)}\left(K_{0}-z^{*}\right)^{-1}\right]^{3} W_{\lambda}^{(\gamma)} f\right\|\left\|W_{\lambda}^{(\gamma)} g\right\|, \quad z \in C_{r_{n}}\left(E_{n}\right) .
\end{aligned}
$$

In the same way as above, we can show that

$$
\left\|\left[V^{(\gamma)}\left(K_{0}-z^{*}\right)^{-1}\right]^{3} W_{\lambda}^{(\gamma)} f\right\|=O\left(\lambda^{7 / 4}\right) \quad(\lambda \rightarrow 0) .
$$

By this estimate and Lemma 6.6, we obtain

$$
(-1)^{3}\left\langle f, W_{\lambda}^{(\gamma)}\left[\left(K_{0}-z\right)^{-1} V^{(\gamma)}\right]^{3}(H(\gamma)-z)^{-1} W_{\lambda}^{(\gamma)} g\right\rangle=O\left(\lambda^{2}\right) \quad(\lambda \rightarrow 0) .
$$

Thus (6.16) with $p=1$ holds.

As for proof of (6.16) with $p \geq 2$, we only sketch it. For each $N \in \mathbb{N}$, we introduce the following operators:

$$
\begin{aligned}
& A_{N}:=\sum_{n=0}^{N}(-1)^{n} W_{\lambda}^{(\gamma)}\left(K_{0}-z\right)^{-1}\left[V^{(\gamma)}\left(K_{0}-z\right)^{-1}\right]^{n}, \\
& B_{N}:=(-1)^{N+1} W_{\lambda}^{(\gamma)}\left[V^{(\gamma)}\left(K_{0}-z\right)^{-1}\right]^{N+1}(H(\gamma)-z)^{-1}
\end{aligned}
$$

Then, by (6.17), we have

$$
W_{\lambda}^{(\gamma)}(H(\gamma)-z)^{-1}=A_{N}+B_{N}
$$


Hence

$$
\left\langle f,\left[W_{\lambda}^{(\gamma)}(H(\gamma)-z)^{-1}\right]^{p} W_{\lambda}^{(\gamma)} g\right\rangle=\left\langle f, A_{N}^{p} W_{\lambda}^{(\gamma)} g\right\rangle+R_{N}
$$

where

$$
R_{N}:=\sum_{j=1}^{p} \sum_{C_{k}=A_{N}, B_{N}, k \neq j}\left\langle f, C_{1} \cdots \stackrel{j-\text { th }}{B}_{N} \cdots C_{p} W_{\lambda}^{(\gamma)} g\right\rangle .
$$

Putting

$$
Q_{n}:=(-1)^{n} W_{\lambda}^{(\gamma)}\left(K_{0}-z\right)^{-1}\left[V^{(\gamma)}\left(K_{0}-z\right)^{-1}\right]^{n}, \quad n \in \mathbb{N}
$$

we have

$$
\left\langle f, A_{N}^{p} W_{\lambda}^{(\gamma)} g\right\rangle=\sum_{n_{1}=0}^{N} \cdots \sum_{n_{p}=0}^{N}\left\langle f, Q_{n_{1}} \cdots Q_{n_{p}} W_{\lambda}^{(\gamma)} g\right\rangle .
$$

After some lengthy calculations using the integral representation of $Q_{n}$ and changes of variables, one can show that

$$
\left\langle f, Q_{n_{1}} \cdots Q_{n_{p}} W_{\lambda}^{(\gamma)} g\right\rangle=O\left(\lambda^{\left(p+2+n_{1}+\cdots+n_{p}\right) / 2}\right) \quad(\lambda \rightarrow 0) .
$$

Hence, for all $N \in \mathbb{N}$,

$$
\left\langle f, A_{N}^{p} W_{\lambda}^{(\gamma)} g\right\rangle=O\left(\lambda^{(p+2) / 2}\right) \quad(\lambda \rightarrow 0)
$$

In the same manner as in the estimation of the fourth term on the right hand side of

(6.18), one can prove that, for all $N$ sufficiently large, $R_{N}=O\left(\lambda^{(p+2) / 2}\right)(\lambda \rightarrow 0)$. Thus (6.16) with $p \geq 2$ holds.

\section{Proof of Theorem 6.4}

We need only to estimate the right hand side of (6.7) asymptotically as $\lambda \rightarrow 0$. By Lemma 6.5 , we have

$$
\left\langle\psi_{n, \ell, s}, W_{\lambda}^{(\gamma)} \psi_{n, \ell, s}\right\rangle=4 \pi \gamma\left|\psi_{n, \ell, s}(\mathbf{0})\right|^{2} \lambda+o(\lambda) \quad(\lambda \rightarrow 0) .
$$

We have for all $N \in \mathbb{N}$

$$
\begin{aligned}
\sum_{p=1}^{\infty}(-1)^{p+1}\left[W_{\lambda}^{(\gamma)}(H(\gamma)-z)^{-1}\right]^{p+1}= & \sum_{p=1}^{N}(-1)^{p+1}\left[W_{\lambda}^{(\gamma)}(H(\gamma)-z)^{-1}\right]^{p+1} \\
& +(-1)^{N+2}\left[W_{\lambda}^{(\gamma)}(H(\gamma)-z)^{-1}\right]^{N+1} W_{\lambda}^{(\gamma)} \\
& \times\left(H_{\lambda}(\gamma)-z\right)^{-1} .
\end{aligned}
$$

Hence, putting

$$
F(\lambda):=\sum_{p=1}^{\infty} F_{n, \ell, s}^{(p)}(\lambda)
$$


we have

$$
F(\lambda)=\sum_{p=1}^{N} F_{n, \ell, s}^{(p)}(\lambda)+F_{N}(\lambda)
$$

where

$F_{N}(\lambda):=\frac{(-1)^{N+2}}{2 \pi i} \int_{C_{r_{n}}\left(E_{n}\right)}\left\langle W_{\lambda}^{(\gamma)}\left[\left(H(\gamma)-z^{*}\right)^{-1} W_{\lambda}^{(\gamma)}\right]^{N+1} \psi_{n, \ell, s},\left(H_{\lambda}(\gamma)-z\right)^{-1} \psi_{n, \ell, s}\right\rangle d z$.

By Lemma 6.7, we have

$$
F_{n, \ell, s}^{(p)}(\lambda)=O\left(\lambda^{(p+2) / 2}\right) \quad(\lambda \rightarrow 0)
$$

By the Schwarz inequality, we have

$$
\left|F_{N}(\lambda)\right| \leq \frac{M_{n}}{1-R_{\lambda, n}} \sup _{z \in C_{r_{n}}\left(E_{n}\right)}\left\|W_{\lambda}^{(\gamma)}\left[\left(H(\gamma)-z^{*}\right)^{-1} W_{\lambda}^{(\gamma)}\right]^{N+1} \psi_{n, \ell, s}\right\| .
$$

In the same way as in the proof of Lemma 6.7, we can show that

$$
\left\|W_{\lambda}^{(\gamma)}\left[\left(H(\gamma)-z^{*}\right)^{-1} W_{\lambda}^{(\gamma)}\right]^{N+1} \psi_{n, \ell, s}\right\|=O\left(\lambda^{(2 N+3) / 4}\right) \quad(\lambda \rightarrow 0)
$$

uniformly in $z \in C_{r_{n}}\left(E_{n}\right)$. Since $N \in \mathbb{N}$ is arbitrary, we can take $N$ sufficiently large so that

$$
F(\lambda)=O\left(\lambda^{3 / 2}\right) \quad(\lambda \rightarrow 0)
$$

It is easy to see that

$$
\lim _{\lambda \rightarrow 0} \sum_{p=1}^{\infty} G_{n, \ell, s}^{(p)}(\lambda)=0
$$

Thus (6.13) holds.

In what follows we assume that, for each $n \in \mathbb{N}, \lambda>0$ is sufficiently small so that the assumption of Theorem 6.3 holds. Then, by Theorem 6.4, we have

$$
\Delta E_{n}\left(\ell, s ; \ell^{\prime}, s^{\prime}\right)=4 \pi \gamma\left(\left|\psi_{n, \ell, s}(\mathbf{0})\right|^{2}-\left|\psi_{n, \ell^{\prime}, s^{\prime}}(\mathbf{0})\right|^{2}\right) \lambda+o(\lambda) \quad(\lambda \rightarrow 0) .
$$

This gives the lowest order approximation in $\lambda$ for the energy level shifts of $H(\gamma)$ with respect to the $n$-th energy level.

Using

$$
L_{n}^{1}(0)=n n !, \quad Y_{0}^{0}=\frac{1}{\sqrt{4 \pi}}
$$

we obtain

$$
\left|\psi_{n, \ell, s}(\mathbf{0})\right|^{2}=\left\{\begin{array}{cc}
\frac{1}{\pi}\left(\frac{m \gamma}{\hbar^{2}}\right)^{3} \frac{1}{n^{3}} & ; \ell=0, s=0 \\
0 & ; \ell \geq 1
\end{array}\right.
$$

Hence the following hold: 
(i) If $\ell, \ell^{\prime} \geq 1$, then

$$
\Delta E_{n}\left(\ell, s ; \ell^{\prime}, s^{\prime}\right)=o(\lambda) \quad(\lambda \rightarrow 0)
$$

(ii) If $\ell \geq 1$, then

$$
\Delta E_{n}(0,0 ; \ell, s)=4 \pi \gamma \lambda\left|\psi_{n, 0,0}(\mathbf{0})\right|^{2}+o(\lambda) \quad(\lambda \rightarrow 0)
$$

Formula (6.23) implies that the energy level with $\ell=0$ is higher than that with $\ell \geq 1$ for all sufficiently small $\lambda>0$. This explains qualitatively the experimental result on the orders of the energy level shifts.

To compare the value of $\Delta E_{n}(0,0 ; \ell, s)$ with the experimental one, we take $\lambda=\lambda_{q}$ with $q=-e$ (see (1.3)) and

$$
\omega(\mathbf{k})=|\mathbf{k}|, \quad \hat{\rho}(\mathbf{k})=\frac{1}{\sqrt{(2 \pi)^{3}}} \chi_{\left[\omega_{\min } / \hbar c, \omega_{\max } / \hbar c\right]}(|\mathbf{k}|), \quad \mathbf{k} \in \mathbb{R}^{3},
$$

with constants $\omega_{\min }>0$ and $\omega_{\max }>0$ satisfying $\omega_{\min }<\omega_{\max }$. Then we have

$$
\lambda=\lambda_{-e}=\alpha\left(\frac{\hbar}{m c}\right)^{2} \frac{1}{3 \pi} \log \frac{\omega_{\max }}{\omega_{\min }},
$$

where

$$
\alpha:=\frac{e^{2}}{4 \pi \hbar c} \approx \frac{1}{137}
$$

is the fine structure constant. We remark that $\omega_{\min }$ (resp. $\omega_{\max }$ ) physically means an infrared (resp. ultraviolet) cutoff of the one-photon energy. We also take

$$
\gamma=\frac{Z e^{2}}{4 \pi}
$$

Thus we obtain

$$
\begin{aligned}
\Delta E_{n}(0,0 ; \ell, s) & \approx \alpha^{5} \frac{4}{3 \pi} m c^{2} \frac{Z^{4}}{n^{3}} \log \frac{\omega_{\max }}{\omega_{\min }} \\
& =\frac{8}{3 \pi} \alpha^{3} \operatorname{Ry} \frac{Z^{4}}{n^{3}} \log \frac{\omega_{\max }}{\omega_{\min }} \quad(\alpha \rightarrow 0),
\end{aligned}
$$

where Ry $:=\alpha^{2} m c^{2} / 2$ is 1 rydberg ( - Ry is the ground state energy of the hydrogen atom). If we take $\omega_{\max }=m c^{2}$ (the rest mass energy of the electron) and $\omega_{\min }=17.8 \mathrm{Ry}$, then the right hand side of (6.24) completely coincides with the Bethe's calculation of the Lamb shift (Eq.(11) in [2]). Thus, in the sense described above, the effective Hamiltonian $H_{\lambda}(\gamma)$ with $\lambda=\lambda_{-e}$ and $\gamma=Z e^{2} / 4 \pi$ explains the Lamb shift of the hydrogen-like atom asymptotically in $\alpha$. 


\section{A A General Perturbation Theory}

In this appendix, we present some fundamental facts in a (not necessarily regular) perturbation theory for self-adjoint operators.

Let $A$ be a self-adjoint operator on a complex Hilbert space $\mathcal{H}$ and $\left\{B_{\lambda} \mid 0<\lambda<a\right\}$ ( $a>0$ is a constant) be a family of symmetric operators on $\mathcal{H}$ such that, for all $\lambda \in(0, a)$, $D(A) \subset D\left(B_{\lambda}\right)$. We assume the following:

\section{Hypothesis (A):}

For all $\lambda \in(0, a)$ and some $z_{0} \in \rho(A)$ (the resolvent set of $\left.A\right), B_{\lambda}\left(A-z_{0}\right)^{-1}$ is bounded and

$$
\lim _{\lambda \rightarrow 0}\left\|B_{\lambda}\left(A-z_{0}\right)^{-1}\right\|=0 .
$$

Remark A.1 Hypothesis (A) implies that, for all $z \in \rho(A)$,

$$
\lim _{\lambda \rightarrow 0}\left\|B_{\lambda}(A-z)^{-1}\right\|=0
$$

uniformly in $z$ on each compact set of $\rho(A)$.

It is obvious that the operator

$$
A_{\lambda}:=A+B_{\lambda}
$$

is symmetric for all $\lambda \in(0, a)$ with $D\left(A_{\lambda}\right)=D(A)$.

Lemma A.2 There exists a constant $c_{0} \in(0, a)$ such that, for all $\lambda \in\left(0, c_{0}\right), A_{\lambda}$ is self-adjoint.

Proof. By Hypothesis (A), for every $\varepsilon \in(0,1)$, there exists a constant $c_{0} \in(0, a)$ such that, for all $\lambda \in\left(0, c_{0}\right),\left\|B_{\lambda}\left(A-z_{0}\right)^{-1}\right\|<\varepsilon$. Hence, for all $\psi \in D(A)$, we have

$$
\left\|B_{\lambda} \psi\right\| \leq \varepsilon\left(\|A \psi\|+\left|z_{0}\right|\|\psi\|\right), \quad \lambda \in\left(0, c_{0}\right) .
$$

Therefore, by the Kato-Rellich theorem [11, Theorem X.12], $A_{\lambda}$ is self-adjoint.

We set

$$
\lambda_{0}:=\sup \left\{\lambda \in(0, a) \mid A_{\lambda^{\prime}} \text { is self-adjoint for every } \lambda^{\prime} \in(0, \lambda)\right\} .
$$

Hence, for all $\lambda \in\left(0, \lambda_{0}\right), A_{\lambda}$ is self-adjoint.

Suppose that $A$ has an isolated eigenvalue $E_{0} \in \mathbb{R}$. Let $r$ be a constant satisfying

$$
0<r<\min _{E \in \sigma(A) \backslash\left\{E_{0}\right\}}\left|E-E_{0}\right|
$$

where $\sigma(A)$ is the spectrum of $A$. Then the circle

$$
C_{r}\left(E_{0}\right):=\left\{z \in \mathbb{C}|| z-E_{0} \mid=r\right\}
$$


with center $E_{0}$ and radius $r$ is included in $\rho(A)$.

Let

$$
R_{\lambda}:=\sup _{z \in C_{r}\left(E_{0}\right)}\left\|B_{\lambda}(A-z)^{-1}\right\|
$$

and

$$
\Lambda:=\left\{\lambda \in\left(0, \lambda_{0}\right) \mid R_{\lambda}<1\right\} .
$$

Then it is easy to see that, for all $\lambda \in \Lambda, C_{r}\left(E_{0}\right) \subset \rho\left(A_{\lambda}\right)$ and the following Neumann expansion holds:

$$
\left(A_{\lambda}-z\right)^{-1}=(A-z)^{-1}+\sum_{n=1}^{\infty}(-1)^{n}(A-z)^{-1}\left[B_{\lambda}(A-z)^{-1}\right]^{n}, \quad z \in C_{r}\left(E_{0}\right)
$$

in operator norm, uniformly in $z \in C_{r}\left(E_{0}\right)$.

For a self-adjoint operator $S$ on a Hilbert space, we denote its spectral measure by $E_{S}(\cdot)$. By functional calculus, the orthogonal projection onto the eigenspace of $A$ with eigenvalue $E_{0}$ is given by

$$
E_{A}\left(\left\{E_{0}\right\}\right)=-\frac{1}{2 \pi i} \int_{C_{r}\left(E_{0}\right)}(A-z)^{-1} d z
$$

where the integral on the right hand side is the contour integral along the circle $C_{r}\left(E_{0}\right)$ with anticlockwise orientation.

Let $\lambda \in \Lambda$. Then, by the fact just mentioned above, we have

$$
E_{A_{\lambda}}\left(\left[E_{0}-r, E_{0}+r\right]\right)=-\frac{1}{2 \pi i} \int_{C_{r}\left(E_{0}\right)}\left(A_{\lambda}-z\right)^{-1} d z .
$$

We set

$$
P:=E_{A}\left(\left\{E_{0}\right\}\right), \quad P_{\lambda}:=E_{A_{\lambda}}\left(\left[E_{0}-r, E_{0}+r\right]\right)
$$

and

$$
N_{r}:=r \sup _{z \in C_{r}\left(E_{0}\right)}\left\|(A-z)^{-1}\right\|
$$

Theorem A.3 Let $\lambda \in \Lambda$ and $R_{\lambda}<1 /\left(1+N_{r}\right)$. Suppose that the multiplicity $m\left(E_{0}\right)$ of $E_{0}$ is finite. Then, $A_{\lambda}$ has exactly $m\left(E_{0}\right)$ eigenvalues in the interval $\left(E_{0}-r, E_{0}+r\right)$, counting multiplicities, and $\sigma\left(A_{\lambda}\right) \cap\left(E_{0}-r, E_{0}+r\right)$ consists of only these eigenvalues.

Proof. By the present assumption for $\lambda$ and (A.5), we have

$$
P_{\lambda}=P+\sum_{n=1}^{\infty} Q_{n}(\lambda)
$$

where

$$
Q_{n}(\lambda):=\frac{(-1)^{n+1}}{2 \pi i} \int_{C_{r}\left(E_{0}\right)}(A-z)^{-1}\left[\left(B_{\lambda}(A-z)^{-1}\right]^{n} d z .\right.
$$

It is easy to see that

$$
\left\|Q_{n}(\lambda)\right\| \leq N_{r} R_{\lambda}^{n}
$$


Hence we have $\left\|P_{\lambda}-P\right\| \leq N_{r} R_{\lambda} /\left(1-R_{\lambda}\right)<1$. Therefore, by a general fact [12, p.14, Lemma], $\operatorname{dim} \operatorname{Ran}\left(P_{\lambda}\right)=\operatorname{dim} \operatorname{Ran}(P)=m\left(E_{0}\right)$. Thus the desired result follows.

Finally we consider the case where $E_{0}$ is a simple eigenvalue of $A$, i.e., $m\left(E_{0}\right)=1$. In this case we denote by $\Omega_{0}$ a normalized eigenvector of $A$ with eigenvalue $E_{0}$ :

$$
A \Omega_{0}=E_{0} \Omega_{0}, \quad\left\|\Omega_{0}\right\|=1 .
$$

Corollary A.4 Let $\lambda \in \Lambda, R_{\lambda}<1 /\left(1+N_{r}\right)$ and $m\left(E_{0}\right)=1$. Then, $A_{\lambda}$ has a unique simple eigenvalue $E_{\lambda}$ in the interval $\left(E_{0}-r, E_{0}+r\right)$ with formula

$$
E_{\lambda}=E_{0}+\frac{\left\langle\Omega_{0}, B_{\lambda} \Omega_{0}\right\rangle+\sum_{n=1}^{\infty} c_{n}(\lambda)}{1+\sum_{n=1}^{\infty} d_{n}(\lambda)},
$$

where

$$
\begin{aligned}
c_{n}(\lambda) & :=\frac{(-1)^{n+1}}{2 \pi i} \int_{C_{r}\left(E_{0}\right)} d z\left\langle\Omega_{0},\left[B_{\lambda}(A-z)^{-1}\right]^{n+1} \Omega_{0}\right\rangle, \\
d_{n}(\lambda) & :=\frac{(-1)^{n+1}}{2 \pi i} \int_{C_{r}\left(E_{0}\right)} d z \frac{\left\langle\Omega_{0},\left[B_{\lambda}(A-z)^{-1}\right]^{n} \Omega_{0}\right\rangle}{E_{0}-z}
\end{aligned}
$$

and $\sigma\left(A_{\lambda}\right) \cap\left(E_{0}-r, E_{0}+r\right)=\left\{E_{\lambda}\right\}$. Moreover, a normalized eigenvector of $A_{\lambda}$ with eigenvalue $E_{\lambda}$ is given by

$$
\Omega_{\lambda}=\frac{\Omega_{0}+\sum_{n=1}^{\infty} \Omega_{\lambda, n}}{\sqrt{1+\sum_{n=1}^{\infty} d_{n}(\lambda)}},
$$

where

$$
\Omega_{\lambda, n}:=\frac{(-1)^{n+1}}{2 \pi i} \int_{C_{r}\left(E_{0}\right)} d z(A-z)^{-1}\left[B_{\lambda}(A-z)^{-1}\right]^{n} \Omega_{0} .
$$

Proof. The existence of the simple eigenvalue $E_{\lambda}$ of $A_{\lambda}$ follows from Theorem A.3 with $m\left(E_{0}\right)=1$. Hence we need only to prove (A.13) and (A.14). In the present case, the vector $\hat{\Omega}_{\lambda}:=P_{\lambda} \Omega_{0}$ is an eigenvector of $A_{\lambda}: A_{\lambda} \hat{\Omega}_{\lambda}=E_{\lambda} \hat{\Omega}_{\lambda}$. It is easy to see that the inequality $\left\|P_{\lambda}-P\right\|<1$ implies $P_{\lambda} \Omega_{0} \neq 0$. Hence $\left\langle P_{\lambda} \Omega_{0}, \Omega_{0}\right\rangle \neq 0$. Taking the inner product of the eigenvector equation for $\hat{\Omega}_{\lambda}$ with the vector $\Omega_{0}$, we obtain

$$
E_{\lambda}=E_{0}+\frac{\left\langle B_{\lambda} \Omega_{0}, P_{\lambda} \Omega_{0}\right\rangle}{\left\langle\Omega_{0}, P_{\lambda} \Omega_{0}\right\rangle}
$$

Using (A.10) and $P \Omega_{0}=\Omega_{0}$, we obtain (A.13).

A normalization of $\hat{\Omega}_{\lambda}$ is given by

$$
\Omega_{\lambda}=\frac{P_{\lambda} \Omega_{0}}{\sqrt{\left\langle\Omega_{0}, P_{\lambda} \Omega_{0}\right\rangle}}
$$

which, combined with (A.10) and $P \Omega_{0}=\Omega_{0}$, we see that (A.14) holds. 


\section{Acknowledgment}

This work was supported by the Grant-In-Aid 21540206 for scientific research from Japan Society for the Promotion of Science (JSPS).

\section{References}

[1] A. Arai, An asymptotic analysis and its application to the nonrelativistic limit of the Pauli-Fierz and a spin-boson model, J. Math. Phys. 31 (1990), 2653-2663.

[2] H. A. Bethe, The electromagnetic shift of energy levels, Phys. Rev. 72 (1947), 339341.

[3] J. D. Bjorken and S. D. Drell, Relativistic Quantum Mechanics, McGraw-Hill, New York, 1964.

[4] C. Hainzl and R. Seiringer, Mass renormalization and energy level shift in nonrelativistic QED, Adv. Theor. Math. Phys. 6 (2002), 847-871.

[5] F. Hiroshima, Observable effects and parameterized scaling limits of a model in nonrelativistic quantum electrodynamics, J. Math. Phys. 43 (2002), 1755-1795.

[6] F. Hiroshima, Analysis of ground states of atoms interacting with a quantized radiation field, in Topics in the Theory of Schrödinger Operators (Editors H. Araki and H. Ezawa), pp.145-272, World Scientific, Singapore, 2004,

[7] S. T. Kuroda, Functional Analysis, Kyoritsu-shuppan, Tokyo, 1980 (in Japanese).

[8] H. Margenau and G. M. Murphy, The Mathematics of Physics and Chemistry, 2nd ed. D. van Nostrand Company, Princeton, New Jersey, 1956.

[9] W. Pauli and M. Fierz, Zur Theorie der Emission Langwelliger Lichtquanten, Nuovo Cimento 15 (1938), 167-188.

[10] M. Reed and B. Simon, Methods of Modern Mathematical Physics Vol.I: Functional Analysis, Academic Press, New York, 1972.

[11] M. Reed and B. Simon, Methods of Modern Mathematical Physics Vol.II: Fourier Analysis and Self-adjointness, Academic Press, New York, 1975.

[12] M. Reed and B. Simon, Methods of Modern Mathematical Physics Vol.IV: Analysis of Operators, Academic Press, New York, 1978.

[13] B. Simon, Quantum Mechanics for Hamiltonians Defined as Quadratic Forms, Princeton University Press, Princeton, 1971.

[14] H. Spohn, Dynamics of Charged Particles and Their Radiation Field, Cambridge University Press, Cambridge, 2004. 
[15] T. A. Welton, Some observable effects of the quantum mechanical fluctuations of the electromagnetic filed, Phys. Rev. 74 (1948), 1157-1167. 